\title{
The clustering of galaxies in the completed SDSS-III Baryon Oscillation Spectroscopic Survey: baryon acoustic oscillations in the Fourier space
}

\section{Florian Beutler, ${ }^{1,2 \star}$ Hee-Jong Seo, ${ }^{3}$ Ashley J. Ross, ${ }^{4}$ Patrick McDonald, ${ }^{2}$} Shun Saito, ${ }^{5,6}$ Adam S. Bolton, ${ }^{7,8}$ Joel R. Brownstein, ${ }^{7}$ Chia-Hsun Chuang, ${ }^{9,10}$ Antonio J. Cuesta, ${ }^{11}$ Daniel J. Eisenstein, ${ }^{12}$ Andreu Font-Ribera, ${ }^{2,5}$ Jan Niklas Grieb, ${ }^{13,14}$ Nick Hand, ${ }^{15}$ Francisco-Shu Kitaura, ${ }^{10}$ Chirag Modi, ${ }^{16}$ Robert C. Nichol, ${ }^{1}$ Will J. Percival, ${ }^{1}$ Francisco Prada, $,{ }^{9}, 17,18$ Sergio Rodriguez-Torres, ${ }^{9,17}$ Natalie A. Roe, ${ }^{2}$ Nicholas P. Ross, ${ }^{19}$ Salvador Salazar-Albornoz, ${ }^{13,14}$ Ariel G. Sánchez, ${ }^{14}$ Donald P. Schneider, ${ }^{20,21}$ Anže Slosar, ${ }^{22}$ Jeremy Tinker, ${ }^{23}$ Rita Tojeiro, ${ }^{24}$ Mariana Vargas-Magaña ${ }^{25}$ and Jose A. Vazquez ${ }^{22}$

Affiliations are listed at the end of the paper

Accepted 2016 September 14. Received 2016 August 28; in original form 2016 July 11

\begin{abstract}
We analyse the baryon acoustic oscillation (BAO) signal of the final Baryon Oscillation Spectroscopic Survey (BOSS) data release (DR12). Our analysis is performed in the Fourier space, using the power spectrum monopole and quadrupole. The data set includes 1198006 galaxies over the redshift range $0.2<z<0.75$. We divide this data set into three (overlapping) redshift bins with the effective redshifts $z_{\text {eff }}=0.38,0.51$ and 0.61 . We demonstrate the reliability of our analysis pipeline using $N$-body simulations as well as $\sim 1000$ MultiDarkPatchy mock catalogues that mimic the BOSS-DR12 target selection. We apply density field reconstruction to enhance the $\mathrm{BAO}$ signal-to-noise ratio. By including the power spectrum quadrupole we can separate the line of sight and angular modes, which allows us to constrain the angular diameter distance $D_{\mathrm{A}}(z)$ and the Hubble parameter $H(z)$ separately. We obtain two independent 1.6 and 1.5 per cent constraints on $D_{\mathrm{A}}(z)$ and 2.9 and 2.3 per cent constraints on $H(z)$ for the low $\left(z_{\text {eff }}=0.38\right)$ and high $\left(z_{\text {eff }}=0.61\right)$ redshift bin, respectively. We obtain two independent 1 and 0.9 per cent constraints on the angular averaged distance $D_{V}(z)$, when ignoring the Alcock-Paczynski effect. The detection significance of the BAO signal is of the order of $8 \sigma$ (post-reconstruction) for each of the three redshift bins. Our results are in good agreement with the Planck prediction within $\Lambda$ cold dark matter. This paper is part of a set that analyses the final galaxy clustering data set from BOSS. The measurements and likelihoods presented here are combined with others in Alam et al. to produce the final cosmological constraints from BOSS.
\end{abstract}

Key words: gravitation-surveys - cosmological parameters-cosmology: observationsdark energy - large-scale structure of Universe.

\section{INTRODUCTION}

The baryon acoustic oscillation (BAO) signal in the distribution of galaxies is an imprint of primordial sound waves that have propagated in the very early Universe through the plasma of tightly

^E-mail: florian.beutler@port.ac.uk coupled photons and baryons (e.g. Peebles \& Yu 1970; Sunyaev $\&$ Zeldovich 1970). The corresponding BAO signal in photons has been observed in the cosmic microwave background (CMB) and has revolutionized cosmology in the last two decades (e.g. Planck Collaboration XIII 2016).

The BAO signal has a characteristic physical scale that represents the distance the sound waves have travelled before the epoch of decoupling. In the distribution of galaxies, the BAO scale is measured 
in angular and redshift coordinates, and this observational metric is related to the physical coordinates through the angular diameter distances and Hubble parameters, which in turn depend on the expansion history of the Universe. Therefore, comparing the BAO scale measured in the distribution of galaxies with the true physical BAO scale, i.e. the sound horizon scale that is independently measured in the $\mathrm{CMB}$, allows us to make cosmological distance measurements to the effective redshift of the distribution of galaxies. With this 'standard ruler' technique one can map the expansion history of the Universe (e.g. Hu \& White 1996; Blake \& Astrophys 2003; Eisenstein 2003; Hu \& Haiman 2003; Seo \& Eisenstein 2003; Linder 2005).

While the BAO feature itself can be isolated from the broadband shape of any galaxy clustering statistic quite easily due to its distinct signature, it is still subject to several observational and evolutionary non-linear effects that damp and shift the BAO feature, thereby biasing such a measurement if ignored (e.g. Meiksin, White, \& Peacock 1999; Seo \& Eisenstein 2005; Crocce \& Scoccimarro 2006; Matsubara 2008b; Taruya et al. 2009; Seo et al. 2010; Mehta et al. 2011). In redshift space, the signal-to-noise ratio of the power spectrum is boosted along the line of sight due to the linear Kaiser factor $\left(1+\beta \mu^{2}\right)^{2}$ (Kaiser 1987), but also suffers the non-linear redshift-space distortion effects that cause additional smearing of the BAO feature along the line of sight.

The BAO method has been significantly strengthened by Eisenstein et al. (2007b), who showed that non-linear degradation effects are reversible by undoing the displacements of galaxies due to bulk flow that is the very cause of the structure growth and redshiftspace distortions. This density field reconstruction technique has been tested against simulations and adopted in current galaxy survey data analyses (e.g. Padmanabhan et al. 2012). In this paper, we will apply this technique.

The galaxy BAO signal was first detected in the Sloan Digital Sky Survey (SDSS)-luminous red galaxies (LRGs; Eisenstein et al. 2005) and 2dF Galaxy Redshift Survey (2dFGRS; Percival et al. 2001; Cole et al. 2005) samples. The WiggleZ survey extended these early detections to higher redshifts (Blake et al. 2011; Kazin et al. 2014), while the 6dF Galaxy Survey (6dFGS) measured the BAO signal at $z=0.1$ (Beutler et al. 2011). Recently the BAO detection in the SDSS main sample at $z=0.15$ was reported in Ross et al. (2015). The first analysis of the Baryon Oscillation Spectroscopic Survey (BOSS) data set in DR9 (Anderson et al. 2012) presented a 1.7 per cent constraint on the angular averaged distance to $z=0.57$, which has been improved to 1 per cent with DR11 (Anderson et al. 2014). The LOWZ sample of BOSS has been used in Tojeiro et al. (2013) to obtain a 2 per cent distance constraint.

While the BAO technique has now been established as the standard tool for cosmology, the anisotropic Fourier-space analysis has been difficult to implement because of the treatment of the window function. To simplify the window function treatment it often has been assumed that the window function is isotropic, which simplifies its treatment considerably. However, the window functions of most galaxy surveys are anisotropic and can introduce anisotropies by redistributing power between the multipoles and potentially bias cosmological measurements. The first self-consistent Fourier-space analysis that does not put such assumptions on the window function was presented in Beutler et al. (2013) using BOSS-DR11, which focused on constraining redshift-space distortions and the Alcock-Paczynski effect. Here we follow this earlier analysis with a few modifications and present a BAO-only analysis, marginalizing over the broad-band power spectrum shape. Our companion paper (Beutler et al. 2016, hereafter B16) goes beyond BAO, studying the additional cosmological information of redshift-space distortions.

This paper uses the combined data from BOSS-LOWZ and BOSS-CMASS, covering the redshift range from $z=0.2$ to 0.75 . The data set also includes additional data from the so-called 'early regions' that have not been included before (see Alam et al. 2016 for details). BAO measurements obtained using the monopole and quadrupole correlation function are presented in Ross et al. (2016), while Vargas-Magaña et al. (2016) diagnose the level of theoretical systematic uncertainty in the BOSS BAO measurements. Measurements of the rate of structure growth from the Redshift-space distortions signal are presented in Beutler et al. (2016), Grieb et al. (2016), Sanchez et al. (2016) and Satpathy et al. (2016). Alam et al. (2016) combine the results of these seven papers (including this work) into a single likelihood that can be used to test cosmological models.

The paper is organized as follows. In Section 2, we introduce the BOSS DR12 data set. In Section 3, we present our anisotropic power spectrum estimator, followed by a description of our window function treatment in Section 4. In Section 5, we present the MultiDark-Patchy mock catalogues that are used to obtain a covariance matrix, and in Section 6, we discuss the technique of density field reconstruction. In Section 7, we introduce our power spectrum model, which we test using $N$-body simulations and the MultiDarkPatchy mock catalogues in Section 8. In Section 9, we present the data analysis, followed by a discussion of the results in Section 10. We conclude in Section 11.

The fiducial cosmological parameters that are used to convert the observed angles and redshifts into comoving coordinates and to generate linear power spectrum models as input for the power spectrum templates, follow a flat $\Lambda$ cold dark matter $(\Lambda \mathrm{CDM})$ model with $\Omega_{\mathrm{m}}=0.31, \Omega_{\mathrm{b}} h^{2}=0.022, h=0.676, \sigma_{8}=0.824, n_{\mathrm{s}}=0.96$, $\sum m_{v}=0.06 \mathrm{eV}$ and $r_{\mathrm{s}}^{\mathrm{fid}}=147.78 \mathrm{Mpc}$.

\section{THE BOSS DR12 DATA SET}

In this analysis we use the final data release (DR12) of the BOSS data set. The BOSS survey is part of SDSS-III (Eisenstein et al. 2011; Dawson et al. 2013) and used the SDSS multifibre spectrographs (Bolton et al. 2012; Smee et al. 2013) to measure spectroscopic redshifts of 1198006 million galaxies. The galaxies were selected from multicolour SDSS imaging (Fukugita et al. 1996; Gunn et al. 1998, 2006; Smith et al. 2002; Doi et al. 2010; Reid et al. 2015) over $10252 \mathrm{deg}^{2}$ divided in two patches on the sky and cover a redshift range of $0.2-0.75$. In our analysis we split this redshift range into three overlapping redshift bins defined by $0.2<z$ $<0.5,0.4<z<0.6$ and $0.5<z<0.75$ with the effective redshifts $z_{\text {eff }}=0.38,0.51$ and $0.61,{ }^{1}$ respectively.

We include three different incompleteness weights to account for shortcomings of the BOSS data set (see Ross et al. 2012a; Anderson et al. 2014 for details): a redshift failure weight, $w_{\mathrm{rf}}$, a fibre collision weight, $w_{\mathrm{fc}}$ and a systematics weight, $w_{\text {sys }}$, which is a combination of a stellar density weight and a seeing condition weight. Each galaxy is thus counted as

$w_{\mathrm{c}}=\left(w_{\mathrm{rf}}+w_{\mathrm{fc}}-1\right) w_{\text {sys }}$.

More details about these weights and their effect on the DR12 sample can be found in Ross et al. (2016).

\footnotetext{
${ }^{1}$ The effective redshifts are calculated as the weighted average over all galaxies (see e.g. equation 67 in Beutler et al. 2013).
} 


\section{THE POWER SPECTRUM ESTIMATOR}

We employ the fast Fourier transform (FFT) based anisotropic power spectrum estimator suggested by Bianchi et al. (2015) and Scoccimarro (2015). This method divides the power spectrum estimate in its spatial components that can then be calculated using 3D Fourier transforms. While this technique requires multiple FFTs, it still provides the computational complexity of $\mathcal{O}(N \log (N))$, significantly faster than a straight forward pair counting analysis that would result in $\mathcal{O}\left(N^{2}\right)$, where $N$ is the number of cells in the 3D Cartesian grid in which the data and random galaxies are binned.

The first two non-zero power spectrum multipoles can be calculated as (Feldman, Kaiser \& Peacock 1993; Yamamoto et al. 2006)

$$
\begin{aligned}
& P_{0}(\boldsymbol{k})=\frac{1}{2 A}\left[F_{0}(\boldsymbol{k}) F_{0}^{*}(\boldsymbol{k})-S\right], \\
& P_{2}(\boldsymbol{k})=\frac{5}{4 A} F_{0}(\boldsymbol{k})\left[3 F_{2}^{*}(\boldsymbol{k})-F_{0}^{*}(\boldsymbol{k})\right],
\end{aligned}
$$

with

$$
\begin{aligned}
F_{0}(\boldsymbol{k})= & A_{0}(\boldsymbol{k}), \\
F_{2}(\boldsymbol{k})= & \frac{1}{k^{2}}\left[k_{x}^{2} B_{x x}+k_{y}^{2} B_{y y}+k_{z}^{2} B_{z z}\right. \\
& \left.+2\left(k_{z} k_{y} B_{x y}+k_{x} k_{z} B_{x z}+k_{y} k_{z} B_{y z}\right)\right] .
\end{aligned}
$$

Following Bianchi et al. (2015) and Scoccimarro (2015) we can write

$A_{0}(\boldsymbol{k})=\int \mathrm{d} \boldsymbol{r} D(\boldsymbol{r}) \mathrm{e}^{\mathrm{i} \boldsymbol{k} \cdot \boldsymbol{r}}$,

$B_{x y}(\boldsymbol{k})=\int \mathrm{d} \boldsymbol{r} \frac{r_{x} r_{y}}{|\boldsymbol{r}|^{2}} D(\boldsymbol{r}) \mathrm{e}^{\mathrm{i} \boldsymbol{k} \cdot \boldsymbol{r}}$,

which allows these quantities to be calculated with FFTs. The overdensity field $D$ is defined on a 3D Cartesian grid $r$ :

$D(\boldsymbol{r})=G(\boldsymbol{r})-\alpha^{\prime} R(\boldsymbol{r})$,

where $G(\boldsymbol{r})$ represents the number of data galaxies at $\boldsymbol{r}$ and $R(\boldsymbol{r})$ is the number of random galaxies at $\boldsymbol{r}$. The normalization of the random field is given by $\alpha^{\prime}=N_{\text {gal }}^{\prime} / N_{\text {ran }}^{\prime}$, where $N_{\text {gal }}^{\prime}$ and $N_{\text {ran }}^{\prime}$ are the total number of weighted data and random galaxies, respectively. The normalization in equations (2) and (3) is

$A=\alpha^{\prime} \sum_{i}^{N_{\mathrm{ran}}} n_{\mathrm{g}}^{\prime}\left(\boldsymbol{r}_{i}\right) w_{\mathrm{FKP}}^{2}\left(\boldsymbol{r}_{i}\right)$,

where $n_{\mathrm{g}}^{\prime}$ is the weighted galaxy density. The shot noise term is only relevant for the monopole and is given by

$$
\begin{aligned}
S= & \sum_{i}^{N_{\mathrm{gal}}}\left[f_{\mathrm{c}} w_{\mathrm{c}}\left(\boldsymbol{x}_{i}\right) w_{\mathrm{sys}}\left(\boldsymbol{x}_{i}\right) w_{\mathrm{FKP}}^{2}\left(\boldsymbol{x}_{i}\right)\right. \\
& \left.+\left(1-f_{\mathrm{c}}\right) w_{\mathrm{c}}^{2}\left(\boldsymbol{x}_{i}\right) w_{\mathrm{FKP}}^{2}\left(\boldsymbol{x}_{i}\right)\right] \\
& +\alpha^{\prime 2} \sum_{i}^{N_{\mathrm{ran}}} w_{\mathrm{FKP}}^{2}\left(\boldsymbol{x}_{i}\right)
\end{aligned}
$$

where $f_{\mathrm{c}}$ is the probability of the fibre collision correction being successful, which we set to 0.5 based on the study by Guo, Zehavi $\&$ Zheng (2012). Even though this definition of the shot noise deviates from the one used in Beutler et al. (2013), the difference does not actually impact our analysis since we marginalize over any residual shot noise (see Section 7). The signal-to-noise ratio weight in the equation above is defined as

$w_{\mathrm{FKP}}=\frac{1}{1+\frac{n_{\mathrm{g}}^{\prime}(z)}{w_{\mathrm{sys}}} 10000}$.

The final power spectrum is then calculated as the average over spherical $k$-space shells:

$P_{\ell}(k)=\left\langle P_{\ell}(\boldsymbol{k})\right\rangle=\frac{1}{N_{\text {modes }}} \sum_{k-\frac{\Delta k}{2}<|\boldsymbol{k}|<k+\frac{\Delta k}{2}} P_{\ell}(\boldsymbol{k})$,

where $N_{\text {modes }}$ is the number of $\boldsymbol{k}$ modes in that shell. In our analysis we chose $\Delta k=0.01 h \mathrm{Mpc}^{-1}$, because this value is significantly smaller than the BAO scale $\sim 0.06 h \mathrm{Mpc}^{-1}$, to not smooth out the BAO signature and about twice as large as the fundamental mode, on which scale the modes are correlated due to the window function.

We employ a triangular-shaped cloud method to assign galaxies to the 3D grid and correct for the aliasing effect following Jing (2005). The set-up of our grid implies a Nyquist frequency of $k_{\mathrm{Ny}}=0.6 h \mathrm{Mpc}^{-1}$, twice as large as the largest scale used in our analysis $\left(k_{\max }=0.3 \mathrm{~h} \mathrm{Mpc}^{-1}\right)$ and the expected error on the power spectrum monopole at $k=0.3 h \mathrm{Mpc}^{-1}$ due to aliasing is $<0.1$ per cent (Sefusatti et al. 2016).

The measured power spectrum multipoles for the three redshift bins are presented in Fig. 1 for NGC and in Fig. 2 for the SGC. We decided not to include the hexadecapole in this analysis, since analytic studies have shown that there is very limited additional information on the anisotropic BAO scale carried by the hexadecapole (Ross, Percival \& Manera 2015).

\section{THE SURVEY WINDOW FUNCTION}

The survey mask is defined as the multiplicative term that turns the Poisson sampled galaxy density field in the observed galaxy density field. In the Fourier space this multiplicative term becomes a convolution. The broad extent of the window function in the Fourier space makes the convolution computationally expensive. Conversely, applying the window function in configuration space is easy and straightforward. Here we follow a method suggested by Wilson et al. (2015) which can be summarized in three steps.

(i) Calculate the model power spectrum multipoles and Fourier transform them to obtain the correlation function multipoles $\xi_{L}^{\text {model }}(s)$.

(ii) Calculate the 'convolved' correlation function multipoles $\hat{\xi}_{\ell}^{\text {model }}(s)$ by multiplying the correlation function with the window function multipoles.

(iii) Conduct 1D FFTs (FFTlog; Hamilton 2000) to transform the convolved correlation function multipoles back into the Fourier space to obtain the convolved power spectrum multipoles, $\hat{P}_{\ell}^{\text {model }}(k)$. This result becomes our model to be compared with the observed power spectrum multipoles.

In Wilson et al. (2015), the formalism following these three steps is derived within the global plane parallel approximations, meaning that a global line of sight, $\hat{\eta}$, is defined for all galaxies in the sample. B16 demonstrates that this method can be derived within the local plane parallel approximation, which means that it is applicable to wide angle surveys such as BOSS. Here we will summarize the formalism and refer to B16 for more details. 

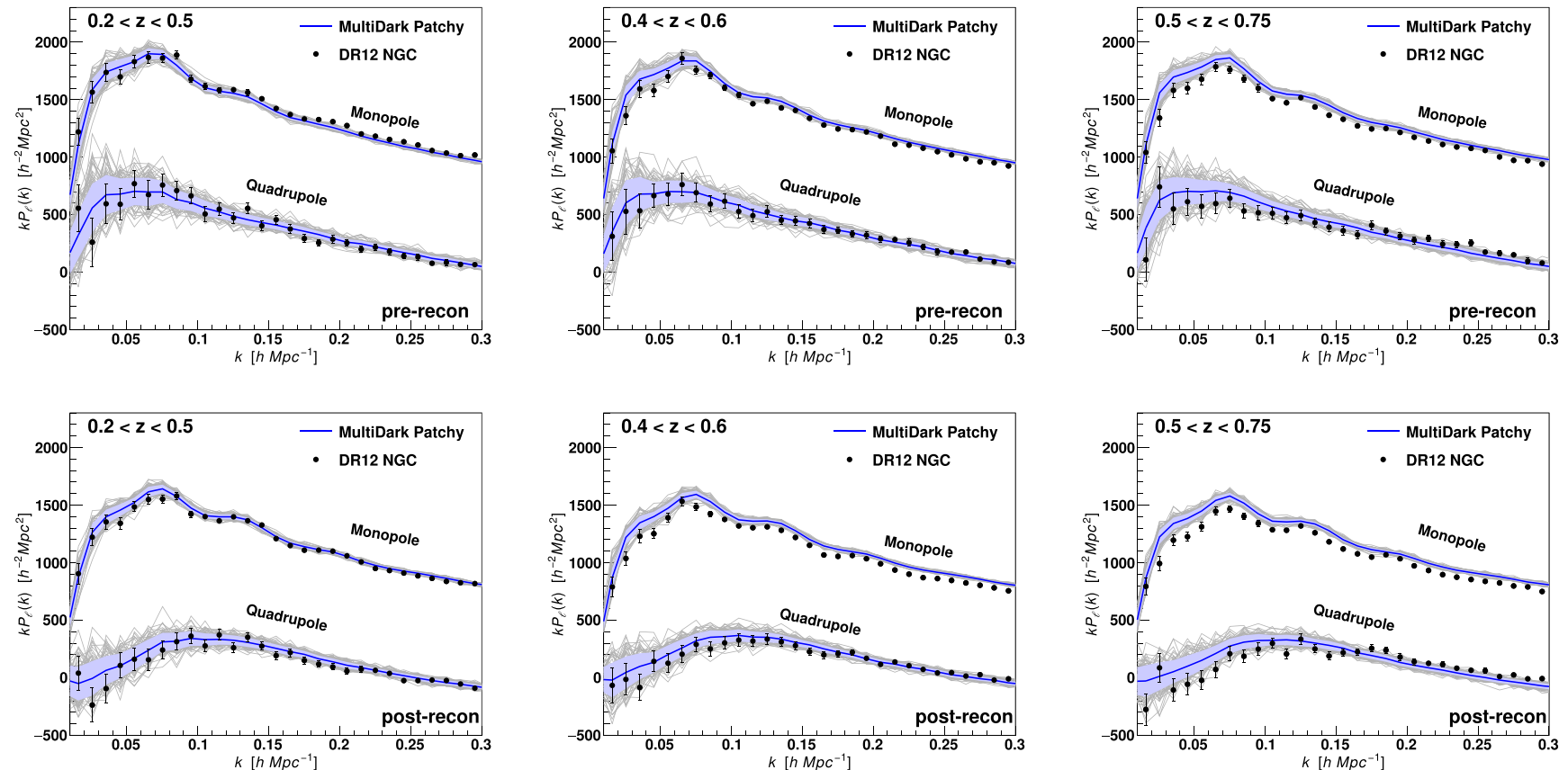

Figure 1. BOSS DR12 power spectra in the North Galactic Cap (NGC) for the three redshift bins used in this analysis. The panels in the top row show the power spectra before density field reconstruction, while the bottom row displays the power spectra after density field reconstruction. The blue line indicates the mean of the 2045 (pre-recon) and 996 (post-recon) MultiDark-Patchy mock catalogues, while the blue shaded area shows the rms between them. The errors on the data points are the diagonal of the covariance matrix.
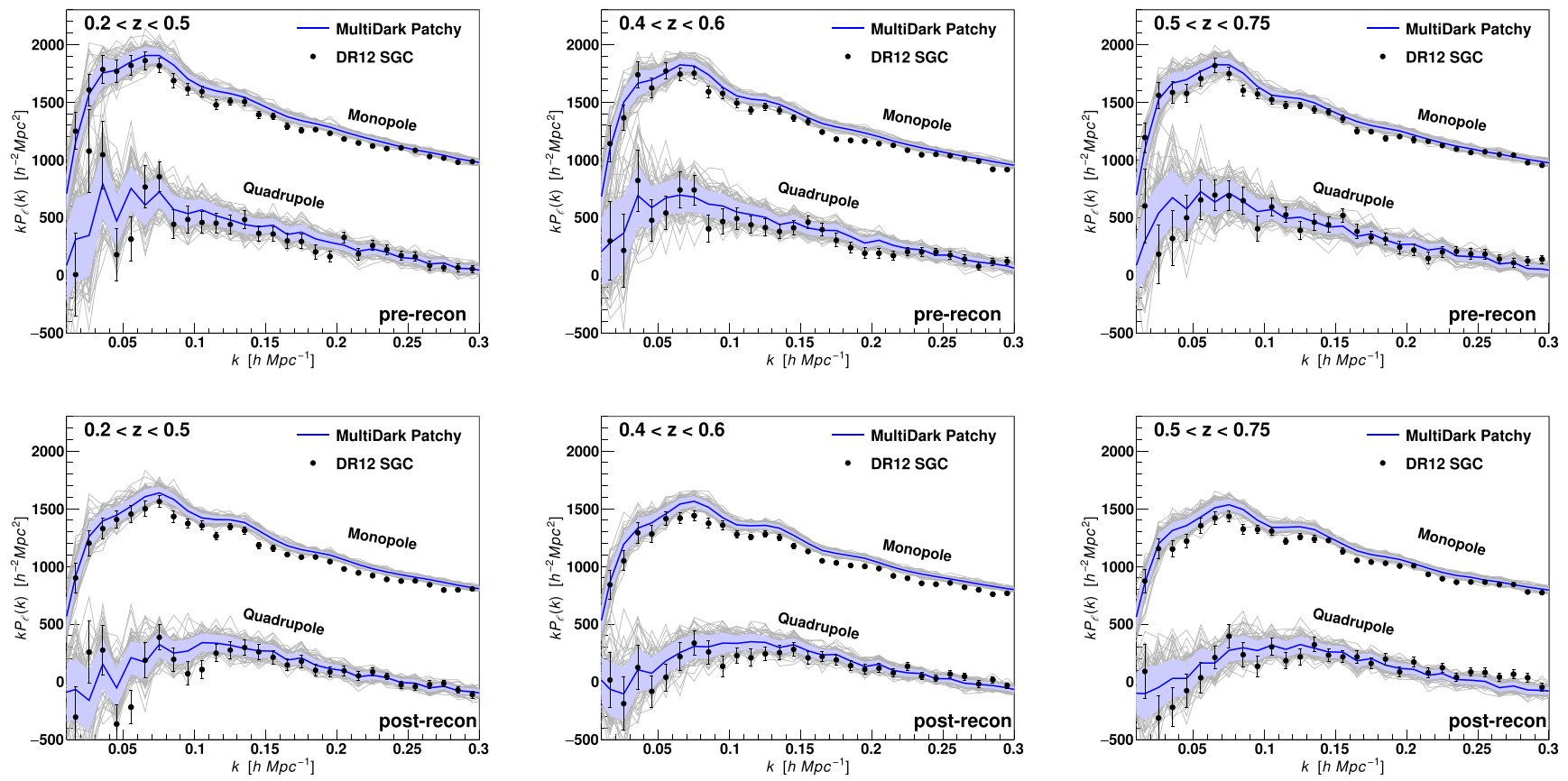

Figure 2. BOSS DR12 power spectra in the South Galactic Cap (SGC) for the three redshift bins used in this analysis. The panels in the top row show the power spectra before density field reconstruction, while the bottom row displays the power spectra after density field reconstruction. The blue line indicates the mean of the 2048 (pre-recon) and 999 (post-recon) MultiDark-Patchy mock catalogues, while the blue shaded area shows the rms between them. The errors on the data points are the diagonal of the covariance matrix. 

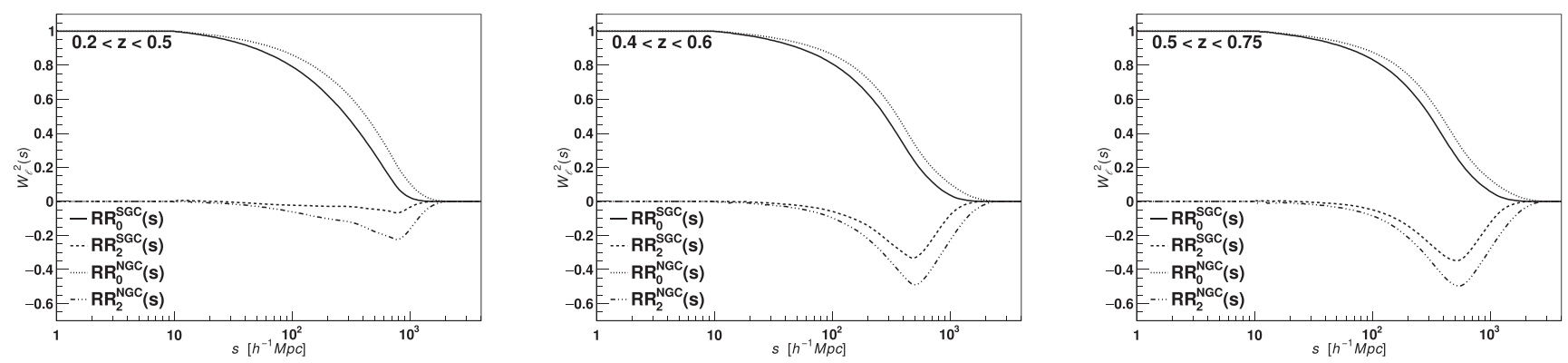

Figure 3. Window function monopole and quadrupole for the three redshift bins of BOSS DR12 as given in equation (18) and used for the convolved correlation functions in equations (19) and (20). As expected, the NGC window function extends to larger scales, because of the larger volume of the NGC compared to the SGC.

The convolved correlation function multipoles can be expressed as

$\hat{\xi}_{\ell}(s)=(2 \ell+1) \sum_{L} \xi_{L}(s) \sum_{p} \frac{1}{2 p+1} W_{p}^{2}(s) a_{L p}^{\ell}$,

with the window function multipoles $W_{p}^{2}(s)$ :

$W_{p}^{2}(s)=\frac{2 p+1}{2} \int \mathrm{d} \mu_{s} \int \mathrm{d} \boldsymbol{x}_{1} W\left(\boldsymbol{x}_{1}\right) W\left(\boldsymbol{x}_{1}+\boldsymbol{s}\right) \mathcal{L}_{p}\left(\mu_{s}\right)$,

where $\mathcal{L}_{\ell}$ is the Legendre polynomial of order $\ell, s=x_{2}-x_{1}$ is the pair separation vector and $\mu_{s}$ is the cosine angle of the separation vector relative to the line of sight, i.e. $\mu_{s}=\hat{\boldsymbol{s}} \cdot \hat{\boldsymbol{x}}_{h}$. To calculate the coefficients $a_{L p}^{\ell}$ we use

$\mathcal{L}_{\ell} \mathcal{L}_{p}=\sum_{t} a_{p t}^{\ell} \mathcal{L}_{t}$

to multiply the polynomial expressions for the Legendre polynomials on the left and apply

$\mu^{n}=\sum_{\ell=n,(n-1), \ldots} \frac{(2 \ell+1) n ! \mathcal{L}_{\ell}\left(\mu_{s}\right)}{2^{(n-\ell) / 2}\left(\frac{1}{2}(n-\ell)\right) !(\ell+n+1) ! !}$.

The convolved power spectrum multipoles are given by

$\hat{P}_{\ell}(k)=4 \pi i^{\ell} \int \mathrm{d} s s^{2} \hat{\xi}_{\ell}(s) j_{\ell}(s k)$.

For any real survey data set the window function is calculated from the random pair counts $R R\left(s, \mu_{s}\right)$ as

$W_{\ell}^{2}(s) \propto \sum_{x_{1}} \sum_{x_{2}} R R\left(s, \mu_{s}\right) \mathcal{L}_{\ell}\left(\mu_{s}\right)$,

with the normalization $W_{0}^{2}(s \rightarrow 0)=1$.

We are interested in the monopole and quadrupole power spectra and therefore, in equation (17), the convolved correlation function multipoles relevant for our analysis are given by

$$
\begin{gathered}
\hat{\xi}_{0}(s)=\xi_{0} W_{0}^{2}+\frac{1}{5} \xi_{2} W_{2}^{2}+\cdots \\
\hat{\xi}_{2}(s)=\xi_{0} W_{2}^{2}+\xi_{2}\left[W_{0}^{2}+\frac{2}{7} W_{2}^{2}\right] \\
+\cdots
\end{gathered}
$$

where we ignored all terms beyond the quadrupole $\xi_{\ell}$. In B16 we find that the hexadecapole contribution to the monopole and quadrupole due to the window function effect can be neglected. The two different window function multipoles included in the equations above are shown in Fig. 3. We assume that the window function is the same for pre- and post-reconstruction.

We account for the integral constraint bias by correcting the model power spectrum as

$P_{\ell}^{\text {ic-corrected }}(k)=\hat{P}_{\ell}(k)-P_{0}(0) W_{\ell}^{2}(k)$,

where the window functions $W(k)$ can be obtained from $W_{\ell}(s)$ defined in equation (18) as

$W_{\ell}^{2}(k)=4 \pi \int \mathrm{d} s s^{2} W_{\ell}^{2}(s) j_{\ell}(s k)$

The integral constraint correction in BOSS only affects modes $k \lesssim$ $0.005 h \mathrm{Mpc}^{-1}$ and does not affect any of the results in this analysis.

\section{MOCK CATALOGUES}

To derive a covariance matrix for the power spectrum monopole and quadrupole we use the MultiDark-Patchy mock catalogues (Kitaura, Yepes \& Prada 2014; Kitaura et al. 2016). These mock catalogues have been produced using approximate gravity solvers and analytical-statistical biasing models. The catalogues have been calibrated to a $N$-body-based reference sample with higher resolution. The reference catalogue is extracted from one of the BigMultiDark simulations (Klypin et al. 2016), which was performed using GADGET-2 (Springel 2005) with $3840^{3}$ particles on a volume of $\left(2.5 h^{-1} \mathrm{Mpc}\right)^{3}$ assuming a $\Lambda \mathrm{CDM}$ cosmology with $\Omega_{\mathrm{M}}=0.307115, \Omega_{\mathrm{b}}=0.048206, \sigma_{8}=0.8288, n_{\mathrm{s}}=0.9611$ and $\mathrm{a}$ Hubble constant of $H_{0}=67.77 \mathrm{~km} \mathrm{~s}^{-1} \mathrm{Mpc}^{-1}$.

Halo abundance matching is used to reproduce the observed BOSS two- and three-point clustering measurements (RodriguezTorres et al. 2016). This technique is applied to different redshift bins to reproduce the BOSS DR12 redshift evolution. These mock catalogues are combined into light cones, also accounting for the selection effects and survey mask of the BOSS survey. In total we have 2045 mock catalogues available for the NGC and 2048 mock catalogues for the SGC. The BAO reconstruction procedure (Eisenstein et al. 2007b) has only been applied to 996 NGC catalogues and 999 SGC catalogues.

The mean power spectrum multipoles for the MultiDark-Patchy mock catalogues are shown in Fig. 1 for the NGC and in Fig. 2 for the SGC together with the BOSS measurements (black data points). The mock catalogues closely reproduce the data power spectrum multipoles for the entire range of wavenumbers relevant for this analysis. 

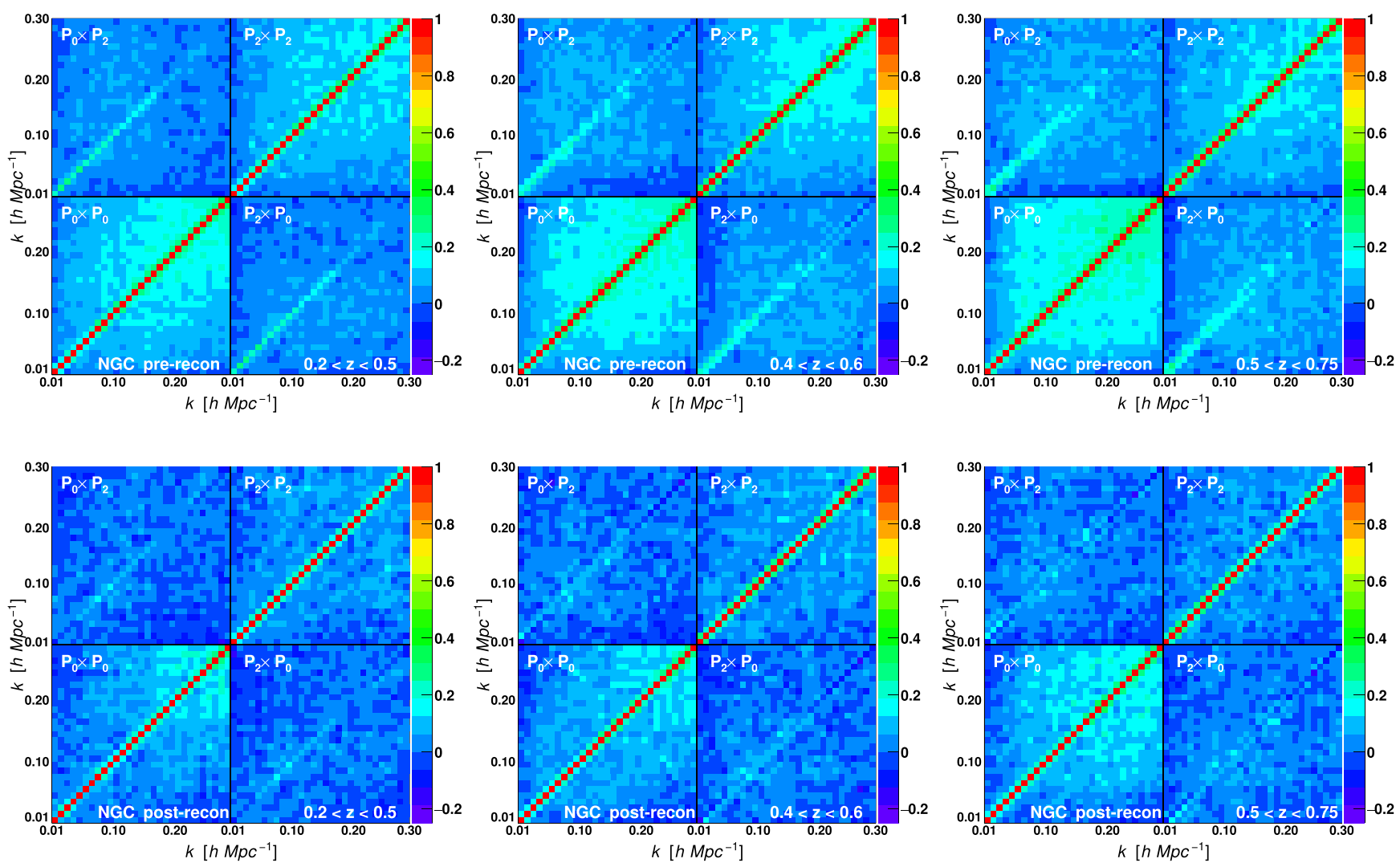

Figure 4. Correlation matrix before (top) and after (bottom) density field reconstruction for the NGC in the three redshift bins used in this analysis. The matrices include the monopole (bottom left-hand corner) and quadrupole (top right-hand corner) as well as their correlation (top left and bottom right). The pre-reconstruction matrices contain 2045 mock catalogues, while the post-reconstruction results contain 996 mock catalogues. The colour indicates the level of correlation, with red corresponding to 100 per cent correlation and magenta corresponding to -25 per cent anticorrelation (there are not many fields lower than -25 per cent). After reconstruction there is less correlation between different $k$ modes and between the multipoles.

\subsection{The covariance matrix}

We can derive a covariance matrix from the set of mock catalogues described in the last section as

$$
\begin{aligned}
C_{x y}= & \frac{1}{N_{\mathrm{s}}-1} \sum_{n=1}^{N_{\mathrm{s}}}\left[P_{\ell, n}\left(k_{i}\right)-\bar{P}_{\ell}\left(k_{i}\right)\right] \\
& \times\left[P_{\ell^{\prime}, n}\left(k_{j}\right)-\bar{P}_{\ell^{\prime}}\left(k_{j}\right)\right],
\end{aligned}
$$

with $N_{\mathrm{s}}$ being the number of mock catalogues. Our covariance matrix at each redshift bin contains the monopole as well as the quadrupole, and the elements of the matrices are given by $(x, y)=\left(\frac{n_{\mathrm{b}} \ell}{2}+i, \frac{n_{\mathrm{b}} \ell^{\prime}}{2}+j\right)$, where $n_{\mathrm{b}}$ is the number of $k$ bins in each multipole power spectrum. Our $k$-binning $\left(\Delta k=0.01 h^{-1} \mathrm{Mpc}\right)$ yields $n_{\mathrm{b}}=29$ for the fitting range $k=0.01-0.30 h^{-1} \mathrm{Mpc}$, and hence the dimensions of the covariance matrices become $58 \times 58$. The mean of the power spectra is defined as

$\bar{P}_{\ell}\left(k_{i}\right)=\frac{1}{N_{\mathrm{s}}} \sum_{n=1}^{N_{\mathrm{s}}} P_{\ell, n}\left(k_{i}\right)$.

Given that the mock catalogues follow the same selection as the data, they incorporate the same window function as we separately match the randoms to each hemisphere.
Figs 4 and 5 present the correlation matrices for BOSS NGC and SGC for the three redshift bins, where the correlation coefficient is defined as

$r_{x y}=\frac{C_{x y}}{\sqrt{C_{x x} C_{y y}}}$.

For each panel in Figs 4 and 5, the lower left-hand corner shows the correlation between bins in the monopole, the upper right-hand corner displays the correlations between the bins in the quadrupole and the upper left-hand corner and lower right-hand corners show the correlation between the monopole and quadrupole. After reconstruction there is less correlation between different $k$ modes. Reconstruction not only sharpens the BAO feature, but also removes some of the correlation between different $k$-modes and between the multipoles, making the covariance matrix more diagonal.

Fig. 6 shows the diagonal elements of the covariance matrix for the monopole and quadrupole power spectrum. We find an error of $\sim 1.5$ percent in the monopole and $\sim 10$ per cent in the quadrupole at $k=0.15 \mathrm{~h} \mathrm{Mpc}^{-1}$. This result represents the most precise measurements of the galaxy power spectrum to date.

Fig. 6 shows the fractional errors for the monopole and quadrupole pre- and post-reconstruction. In the Gaussian limit the fractional errors depend on the number of independent $k$-modes and the shot noise contribution (on small scales). Because the error decreases by the same factor as the power spectrum decreases, the fractional errors of the monopole are almost identical before 

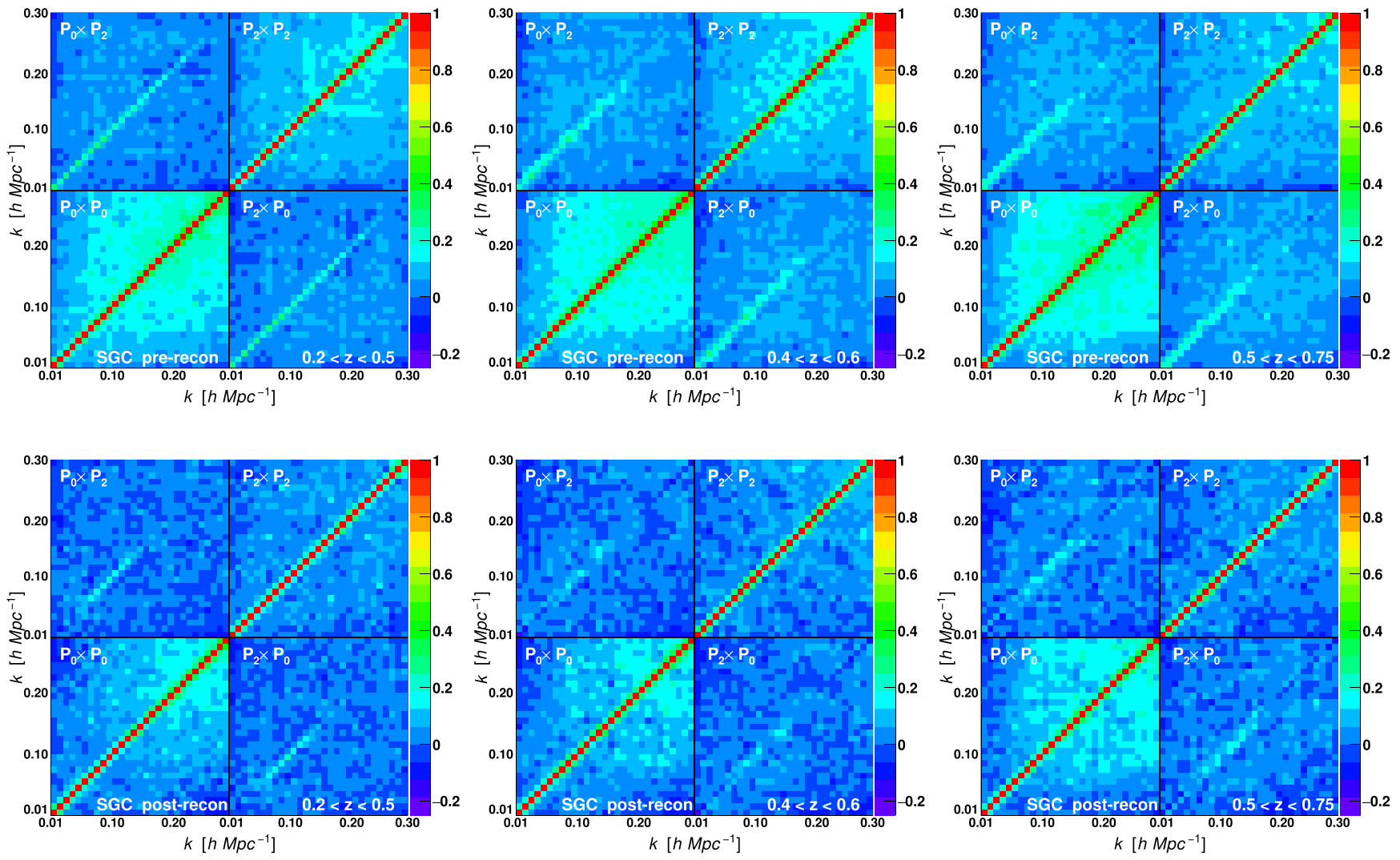

Figure 5. Correlation matrix before (top) and after (bottom) density field reconstruction for the SGC in the three redshift bins used in this analysis. The matrices include the monopole (bottom left-hand corner) and quadrupole (top right-hand corner) as well as their correlation (top left and bottom right). The pre-reconstruction matrices contain 2048 mock catalogues, while the post-reconstruction results contain 999 mock catalogues. The colour indicates the level of correlation, with red corresponding to 100 per cent correlation and magenta corresponding to -25 per cent anticorrelation (there are not many fields lower than -25 per cent). After reconstruction there is less correlation between different $k$ modes and between the multipoles.

and after reconstruction on large scales. Since reconstruction cannot remove the shot noise contribution, the error on small scales cannot decrease significantly, while non-linearity on the shot-noise subtracted monopole is reduced by a small amount. This situation makes the fractional errors of the monopole after reconstruction (dashed lines) slightly larger than the monopole before reconstruction (solid lines) on small scales. The leading contribution to the quadrupole error is produced by the monopole power spectrum, not the quadrupole power spectrum (see appendix of Taruya, Nishimichi \& Saito 2010; Yoo \& Seljak 2015). On small scales, the non-linear effects on the quadrupole decrease after reconstruction, while again the shot noise contribution to the quadrupole error remains the same. As a result, the fractional error on the quadruple on small scales also becomes larger after reconstruction. This result does not contradict the observed improved information content (e.g. Ngan et al. 2012) after reconstruction since the information content accounts for the entire covariance between different modes and different multipoles, which is reduced after reconstruction. Also, the main signal-to-noise ratio improvement from the $\mathrm{BAO}$ reconstruction is produced by the sharpening of the BAO.

\subsection{Inverting the mock covariance matrix}

As the estimated covariance matrix $C$ is inferred from mock catalogues, its inverse, $C^{-1}$, provides a biased estimate of the true inverse covariance matrix, (Hartlap, Simon \& Schneider 2007). To correct for this bias we rescale the inverse covariance matrix as

$C_{i j, \text { Hartlap }}^{-1}=\frac{N_{\mathrm{s}}-n_{\mathrm{b}}-2}{N_{\mathrm{s}}-1} C_{i j}^{-1}$,

where $n_{\mathrm{b}}$ is the number of power spectrum bins. This scaling assumes a Gaussian error distribution and an uncorrelated data vector, which is not strictly true for our data set (see Figs 4 and 5). We therefore produce many random simulations to keep this scaling factor small. For our post-reconstruction case with $n_{\mathrm{b}}=58$ and $N_{\mathrm{s}}=999$ for the SGC ( $N_{\mathrm{s}}=996$ for the NGC) the correction of equation (26) increases the parameter variance by about 6 per cent. With these covariance matrices we can then perform a standard $\chi^{2}$ minimization to find the best-fitting parameters.

\section{DENSITY FIELD RECONSTRUCTION}

The main complication of studying BAO in the distribution of galaxies compared to similar studies in the $\mathrm{CMB}$ arises due to non-linear structure evolution; the damping of the $\mathrm{BAO}$ feature lessens the precision of the BAO measurements, and potential shifts in the BAO scale can introduce a systematic bias on the resulting cosmology. Redshift-space distortions enhance such complications along the line of sight.

Density field reconstruction (Eisenstein et al. 2007b) is a technique to enhance the signal-to-noise ratio of the BAO signature 

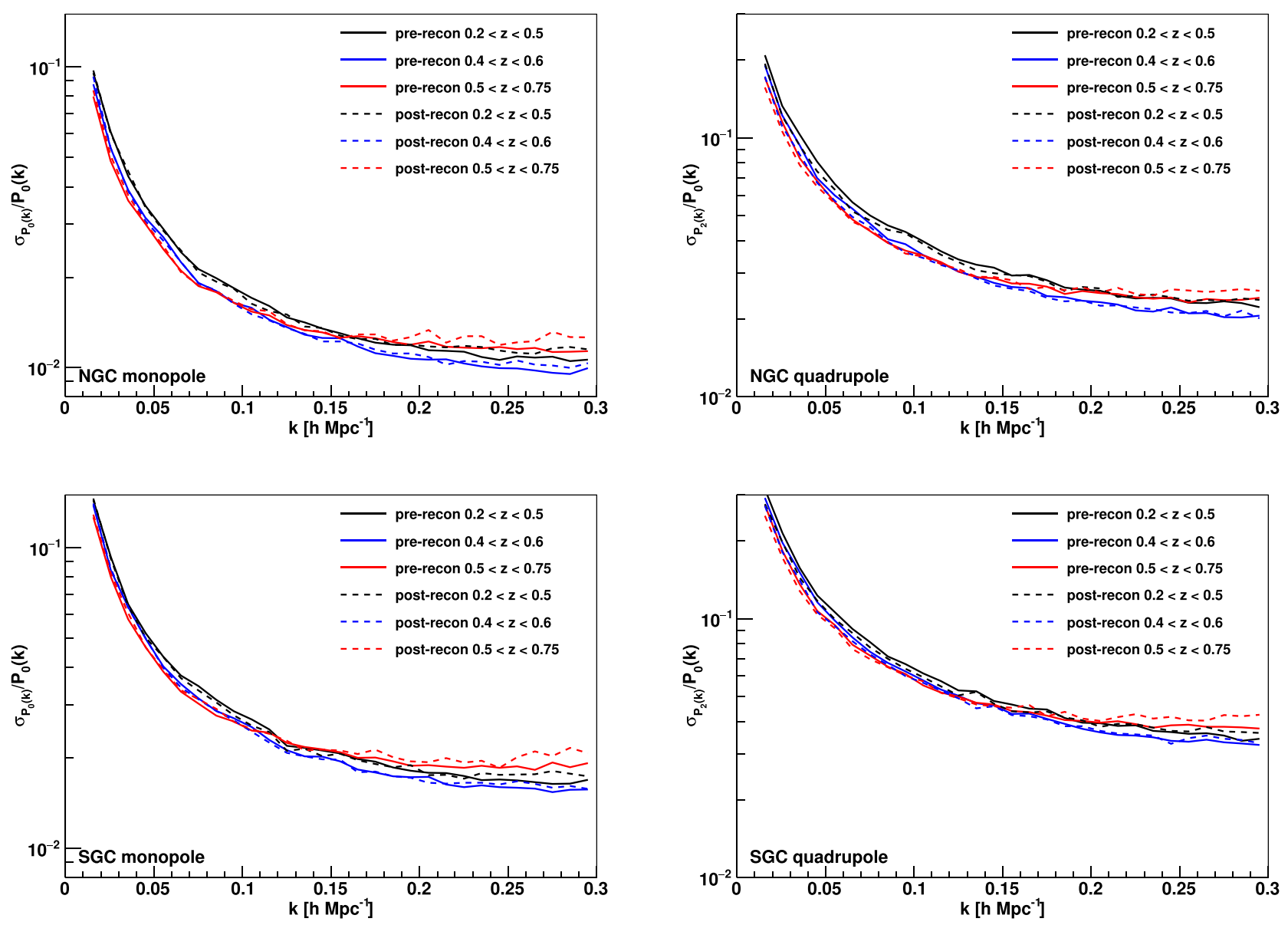

Figure 6. The relative uncertainty of the NGC power spectrum monopole (left) and quadrupole (right) before (solid lines) and after (dashed lines) density field reconstruction. The power spectrum monopole in the denominator does have the shot noise subtracted (we use the monopole in the denominator of the quadrupole plot because the quadrupole is often nearly zero).

by partly undoing non-linear effects of structure formation and redshift-space distortions, i.e. by bringing the information that leaked to the higher order statistics of the galaxy distribution back to the two-point statistic (Schmittfull et al. 2015). The main steps of density field reconstruction are the following.

(i) Estimate the displacement field due to structure growth and redshift-space distortions based on the observed galaxy density field.

(ii) Displace the observed galaxies and a sample of randomly distributed particles with this estimated displacement field.

(iii) Subtract the data and random displaced density fields.

In this analysis we follow the method of Padmanabhan et al. (2012). The observed redshift-space galaxy density field is calculated as

$\delta(s)=\frac{G(s)}{\alpha^{\prime} R(s)}-1$,

where $G$ and $R$ are defined in equation (8). We smooth this field with a Gaussian filter of the form

$S(k)=\exp \left[-\left(k \Sigma_{\text {smooth }}\right)^{2} / 2\right]$,

where we chose $\Sigma_{\text {smooth }}=15 h^{-1} \mathrm{Mpc}$, which is close to the optimal smoothing scale given the signal-to-noise ratio of the BOSS data (Xu et al. 2012; Burden et al. 2014; Seo et al. 2016; VargasMagaña et al. 2015). In linear perturbation theory, the real-space displacement field $\boldsymbol{\Psi}(x)$ is related to the redshift-space density field by

$\nabla \cdot \boldsymbol{\Psi}(\boldsymbol{s})+\beta \nabla \cdot\left(\boldsymbol{\Psi} \cdot \hat{\boldsymbol{s}}_{\|}\right) \hat{\boldsymbol{s}}_{\|}=-\frac{\delta(\boldsymbol{s})}{b}$,

where $\hat{\boldsymbol{s}}_{\text {los }}$ is the unit vector along the line of sight (Nusser \& Davis 1994). Assuming the $\boldsymbol{\Psi}$ is irrotational, we write $\boldsymbol{\Psi}=\nabla \phi$ and solve for the scalar potential $\phi$. To do this, we convert all the derivatives to their finite difference counterparts and solve the resulting linear equation (Padmanabhan et al. 2012). Once $\phi$ is derived, $\boldsymbol{\Psi}$ can be calculated using finite differences.

We then apply the displacement to our galaxies by shifting their line of sight and angular position following

$s_{\|}^{\text {new }}=s_{\|}^{\text {old }}-(1+f) \Psi_{\|}\left(s^{\text {old }}\right)$,

$s_{\perp}^{\text {new }}=s_{\perp}^{\text {old }}-\Psi_{\perp}\left(s^{\text {old }}\right)$,

where we multiply the derived displacement with $(1+f)$ when displacing the galaxies along the line of sight in order to remove linear redshift-space distortions.

The procedure of reconstruction outlined above does rely on a fiducial cosmological model providing the growth rate $f(z)$, needed in equation (30) as well as the bias parameter in equation (29). We refer to Mehta et al. (2011) and Vargas-Magaña et al. (2015) for a detailed study of how these initial assumptions influence the reconstructed $\mathrm{BAO}$ results. 
This procedure leads to a shifted galaxy, $G^{\mathrm{s}}(\boldsymbol{r})$, and shifted random catalogue, $R^{\mathrm{s}}(\boldsymbol{r})$, where the positions of all galaxies are modified based on the estimated displacement field. The overdensity field, $D(\boldsymbol{r})$, required for the power spectrum estimate can be obtained in an analogous way to equation (8) and is given by

$D^{\mathrm{s}}(\boldsymbol{r})=G^{\mathrm{s}}(\boldsymbol{r})-\alpha^{\prime} R^{\mathrm{s}}(\boldsymbol{r})$.

\section{THE POWER SPECTRUM MODEL}

Here we introduce the anisotropic and isotropic power spectrum model used to extract the BAO information by fitting to the measurements. The method used in this paper follows Anderson et al. $(2012,2014)$ with small modifications as discussed in Seo et al. (2016).

\subsection{The anisotropic case}

Our anisotropic power spectrum model is given by

$$
\begin{aligned}
P(k, \mu)= & P_{\mathrm{sm}}(k, \mu) \\
& \times\left[1+\left(O_{\operatorname{lin}}(k)-1\right) \mathrm{e}^{-\left[k^{2} \mu^{2} \Sigma_{\|}^{2}+k^{2}\left(1-\mu^{2}\right) \Sigma_{\perp}^{2}\right] / 2}\right],
\end{aligned}
$$

where $\mu$ is the cosine angle to the line of sight, $O_{\text {lin }}(k)$ represents the oscillatory part of the fiducial linear power spectrum and $P_{\mathrm{sm}}(k$, $\mu)$ is the smooth anisotropic power spectrum. We use two damping scales to model the anisotropic non-linear damping on the BAO feature, one for modes along the line of sight, $\Sigma_{\|}$, and one for modes perpendicular to the line of sight, $\Sigma_{\perp}$. To obtain $O_{\text {lin }}(k)$ we fit the fiducial linear power spectrum, $P_{\text {lin }}(k)$, with Eisenstein \& $\mathrm{Hu}$ (1998) no-Wiggle power spectrum, $P_{\mathrm{nw}}(k)$, together with five polynomial terms and derive a smooth fit, $P_{\mathrm{sm}, \operatorname{lin}}(k)$. The oscillatory part is then given by

$O_{\text {lin }}(k)=\frac{P_{\text {lin }}(k)}{P_{\text {sm }, \text { lin }}(k)}$.

The smooth anisotropic power spectrum, $P_{\mathrm{sm}}(k, \mu)$, is given by

$P_{\mathrm{sm}}(k, \mu)=B^{2}\left(1+\beta \mu^{2} R\right)^{2} P_{\mathrm{sm}, \operatorname{lin}}(k) F_{\mathrm{fog}}\left(k, \mu, \Sigma_{\mathrm{s}}\right)$,

where the parameter $B$ is used to marginalize over the power spectrum amplitude. We set $R=1$ before density field reconstruction and $R=1-\exp \left[-\left(k \Sigma_{\text {smooth }}\right)^{2} / 2\right]$ after reconstruction. Seo et al. (2016) demonstrate that this $R$-term after reconstruction depends on the conventions used in the reconstruction process. The $R$-term we use accounts for the removal of redshift-space distortions on large scales during reconstruction ('Rec-Iso' convention in Seo et al. 2016), and the smoothing scale $\Sigma_{\text {smooth }}=15 h^{-1} \mathrm{Mpc}$ used when deriving the displacement field (see Section 6 for details). The damping term $F_{\text {fog }}\left(k, \mu, \Sigma_{\mathrm{s}}\right)$ due to the non-linear velocity field (finger-of-God) is given by

$F_{\text {fog }}\left(k, \mu, \Sigma_{\mathrm{s}}\right)=\frac{1}{\left(1+k^{2} \mu^{2} \Sigma_{\mathrm{s}}^{2} / 2\right)^{2}}$.

We add extra polynomial terms to marginalize over the angledependent overall shape of the power spectrum. The power spectrum monopole and quadrupole are

$$
\begin{aligned}
& P_{0}(k)=\frac{1}{2} \int_{-1}^{1} P(k, \mu) \mathrm{d} \mu+A_{0}(k), \\
& P_{2}(k)=\frac{5}{2} \int_{-1}^{1} P(k, \mu) \mathcal{L}_{2}(\mu) \mathrm{d} \mu+A_{2}(k),
\end{aligned}
$$

where

$$
\begin{aligned}
& A_{\ell}^{\text {pre-recon }}(k)=\frac{a_{\ell, 1}}{k^{3}}+\frac{a_{\ell, 2}}{k^{2}}+\frac{a_{\ell, 3}}{k}+a_{\ell, 4}+a_{\ell, 5} k, \\
& A_{\ell}^{\text {post-recon }}(k)=\frac{a_{\ell, 1}}{k^{3}}+\frac{a_{\ell, 2}}{k^{2}}+\frac{a_{\ell, 3}}{k}+a_{\ell, 4}+a_{\ell, 5} k^{2} .
\end{aligned}
$$

The decision which polynomial to use is based on the $\Delta \chi^{2}$ achieved by each term. The data prefer a linear polynomial in the pre-recon case and a $k^{2}$ polynomial post-recon leading to a different set of polynomials in the two cases.

Given that the galaxies in the NGC and SGC follow slightly different selections (Alam et al. 2016), we use two separate parameters to describe the clustering amplitude in the two samples: $B_{\mathrm{SGC}}$ and $B_{\mathrm{NGC}}$. In our analysis we fix $\Sigma_{\|}=4 h^{-1} \mathrm{Mpc}$ and $\Sigma_{\perp}=2 h^{-1} \mathrm{Mpc}$ for the post-reconstruction case and $\Sigma_{\|}=8 h^{-1} \mathrm{Mpc}$ and $\Sigma_{\perp}=4 h^{-1} \mathrm{Mpc}$ for the pre-reconstruction case (Eisenstein, Seo \& White 2007a). The exact choice for $\Sigma_{\perp}$ and $\Sigma_{\|}$does not affect our analysis. This approach leads to 14 free nuisance parameters ( $B_{\mathrm{SGC}}, B_{\mathrm{NGC}}, \beta, a_{\ell, 1-5}, \Sigma_{\mathrm{s}}$ ). In Section 7.2 we will introduce the two BAO scale parameters $\alpha_{\perp}$ and $\alpha_{\|}$, which will complete our set of 16 free fitting parameters (we fit the NGC and SGC power spectra simultaneously).

\subsection{The anisotropic standard ruler test}

If the fiducial cosmological parameters used to convert galaxy redshifts into physical distances and angular separations into the physical separations deviate from the true cosmology, the observed BAO scale will deviate from the true BAO scale, i.e. the sound horizon scale $r_{\mathrm{s}}\left(z_{\mathrm{d}}\right)$. In the full (i.e. anisotropic) standard ruler test, we can measure the deviations along the line of sight and perpendicular to the line of sight separately, thereby deriving constraints on the true Hubble parameter and angular diameter distance relative to the fiducial relations. We parametrize the observed BAO scales along and perpendicular to the line of sight relative to the BAO scale in the power spectrum template using following two scaling parameters:

$\alpha_{\|}=\frac{H^{\mathrm{fid}}(z) r_{\mathrm{s}}^{\mathrm{fid}}\left(z_{\mathrm{d}}\right)}{H(z) r_{\mathrm{s}}\left(z_{\mathrm{d}}\right)}$,

$\alpha_{\perp}=\frac{D_{\mathrm{A}}(z) r_{\mathrm{s}}^{\mathrm{fid}}\left(z_{\mathrm{d}}\right)}{D_{\mathrm{A}}^{\mathrm{fid}}(z) r_{\mathrm{s}}\left(z_{\mathrm{d}}\right)}$,

where $H^{\mathrm{fid}}(z)$ and $D_{\mathrm{A}}^{\mathrm{fid}}(z)$ are the fiducial values for the Hubble parameter and angular diameter distance at the effective redshift of the sample, and $r_{\mathrm{s}}^{\mathrm{fid}}\left(z_{\mathrm{d}}\right)$ is the fiducial sound horizon assumed in the template power spectrum. The sound horizon scale $r_{\mathrm{s}}^{\mathrm{fid}}\left(z_{\mathrm{d}}\right)$ is considered here to correct for the fiducial location of the BAO feature assumed in the template. Alternatively we can use the values

$\alpha=\alpha_{\|}^{1 / 3} \alpha_{\perp}^{2 / 3}$,

$\epsilon=\left(\frac{\alpha_{\|}}{\alpha_{\perp}}\right)^{1 / 3}-1$

where $\alpha$ describes an isotropic shift (radial dilation) in the BAO scale and $\epsilon$ captures any anisotropic warping. We will employ both expressions for the rest of this paper.

The true wavenumbers $\left(k_{\|}^{\prime}\right.$ and $\left.k_{\perp}^{\prime}\right)$ are related to the observed wavenumbers by $k_{\|}^{\prime}=k_{\|} / \alpha_{\|}$and $k_{\perp}^{\prime}=k_{\perp} / \alpha_{\perp}$. Transferring this information into scalings for the absolute wavenumber $k=\sqrt{k_{\|}^{2}+k_{\perp}^{2}}$ and the cosine of the angle to the line of sight $\mu$, we can relate the 
true and observed values by

$k^{\prime}=\frac{k}{\alpha_{\perp}}\left[1+\mu^{2}\left(\frac{1}{F^{2}}-1\right)\right]^{1 / 2}$,

$\mu^{\prime}=\frac{\mu}{F}\left[1+\mu^{2}\left(\frac{1}{F^{2}}-1\right)\right]^{-1 / 2}$,

with $F=\alpha_{\|} / \alpha_{\perp}$ (Ballinger, Peacock \& Heavens 1996). The multipole power spectrum, including the BAO radial dilation and warping, can be written as

$P_{\ell}(k)=\left(\frac{r_{\mathrm{s}}^{\mathrm{fid}}}{r_{\mathrm{s}}}\right)^{3} \frac{(2 \ell+1)}{2 \alpha_{\perp}^{2} \alpha_{\|}} \int_{-1}^{1} \mathrm{~d} \mu P_{\mathrm{g}}\left[k^{\prime}(k, \mu), \mu^{\prime}(\mu)\right] \mathcal{L}_{\ell}(\mu)$,

where $\left(\frac{r_{s}^{\text {fid }}}{r_{\mathrm{s}}}\right)^{3} \frac{1}{\alpha_{\perp}^{2} \alpha_{\|}}$accounts for the difference in the cosmic volume in different cosmologies. The ratio of sound horizons is needed to compensate for the sound horizons included in the definitions of the $\alpha$ values. However, since this term degenerates with our free amplitude parameters, it has no effect on our BAO analysis.

\subsection{The isotropic case}

We also constrain the angle average BAO dilation scale using only the monopole power spectrum, which ignores the AlcockPaczynski effect by holding the Alcock-Paczynski shape $D_{\mathrm{A}} H$ fixed at the fiducial shape, while spherically averaging the clustering information (i.e. we are assuming the radial and transverse distance scales to be same). In that case we cannot separately constrain $D_{\mathrm{A}}$ and $H$, but only the radial BAO dilation in a spherically averaged clustering, which is traditionally defined as

$D_{V}(z)=\left[(1+z)^{2} D_{\mathrm{A}}^{2}(z) \frac{c z}{H(z)}\right]^{1 / 3}$.

Our model for the isotropic (monopole only) analysis is a simplified version of the model used in the anisotropic case. Since $\beta$ and $B$ are degenerate when fitting only the monopole power spectrum before reconstruction, we remove the $\left(1+\beta \mu^{2}\right)^{2}$ term. The oscillation damping term simplifies to $\Sigma_{\mathrm{nl}} \sim \sqrt{\left(\Sigma_{\|}^{2}+2 \Sigma_{\perp}^{2}\right) / 3}$, and we remove the $\mu$ dependence in equation (36). We therefore have

$P(k)=P_{\mathrm{sm}}(k)\left[1+\left(O_{\text {lin }}(k)-1\right) \mathrm{e}^{-\left[k^{2} \Sigma_{\mathrm{n}}^{2}\right] / 2}\right]$

and

$P_{\mathrm{sm}}(k)=B^{2} P_{\mathrm{sm}, \operatorname{lin}}(k) F_{\mathrm{fog}}\left(k, \Sigma_{\mathrm{s}}\right)$,

with $P_{\mathrm{sm} \text {, lin }}$ as given in equation (35). The velocity damping term is given by

$F_{\text {fog }}\left(k, \Sigma_{\mathrm{s}}\right)=\frac{1}{\left(1+k^{2} \Sigma_{\mathrm{s}}^{2} / 2\right)^{2}}$.

The effect of the radial dilation of the BAO is included as

$P_{0}(k)=\left(\frac{r_{\mathrm{s}}^{\mathrm{fid}}}{r_{\mathrm{s}}}\right)^{3} \frac{(2 \ell+1)}{2 \alpha^{3}} \int_{-1}^{1} \mathrm{~d} \mu P_{\mathrm{g}}\left(k^{\prime}=k / \alpha, \mu\right) \mathcal{L}_{0}(\mu)$,

where

$\alpha=\frac{D_{V}(z) r_{\mathrm{s}}^{\mathrm{fid}}\left(z_{\mathrm{d}}\right)}{D_{V}^{\mathrm{fid}}(z) r_{\mathrm{s}}\left(z_{\mathrm{d}}\right)}$.

In total, we have 10 free parameters in the isotropic case $\left(B^{\mathrm{NGC}}\right.$, $\left.B^{\mathrm{SGC}}, a_{0,1-5}, \alpha, \Sigma_{\mathrm{nl}}, \Sigma_{\mathrm{s}}\right)$.

We expect the constraint on $\alpha$ in the isotropic case to be tighter than the constraint on $\alpha$ in the anisotropic case, since in the latter, $\alpha$

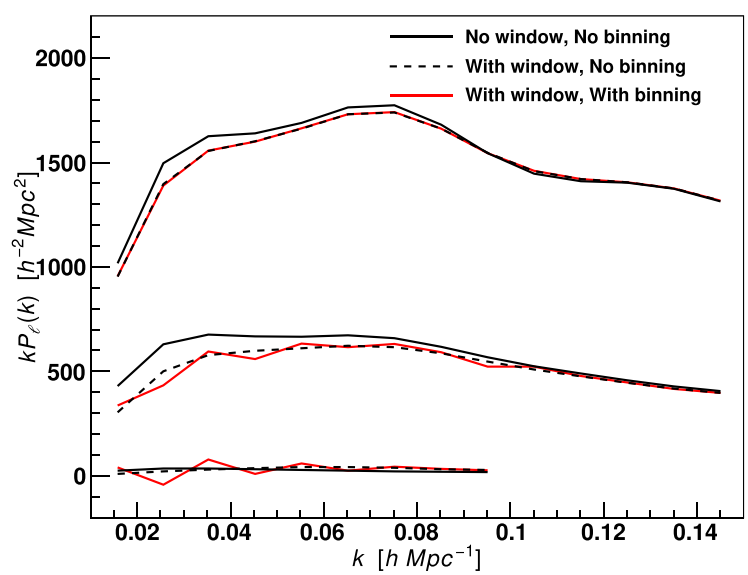

Figure 7. The window function and discreteness effects for the lowest redshift bin in the SGC. The three lines show the raw power spectrum model (black solid line), the same model including the convolution with the window function (black dashed line) and including the discreteness effect of Section 7.4 (red solid line). The SGC in the lowest redshift bin has the smallest volume and therefore both window function and discreteness effects are expected to be the largest in this case.

(in equation 43) is marginalized over the warping effect while in the former analysis it is not. We will consider the anisotropic constraints as our main result, since the anisotropic analysis depends on fewer assumptions. We will show constraints on $D_{V}$ from the isotropic analysis only for comparison.

\subsection{Correction for the irregular $\mu$ distribution}

Because the survey volume is not infinite, the power spectra are estimated on a finite and discrete $k$-space grid. Performing FFTs in a Cartesian lattice makes the angular distribution of the Fourier modes irregular and causes deviation from the isotropic distribution, more so at smaller $k$. As a result, we see small fluctuation-like deviations in the measured power spectrum multipoles that are not caught by the window function, as shown in Fig. 7. The effect is larger for the quadrupole than the monopole since the quadrupole is more sensitive to an anisotropy of the mode distribution. Given that the SGC in the lowest redshift bin has the smallest volume, we expect this effect to be greatest for this case. In this paper, we include this effect in our power spectrum monopole and quadrupole model. When calculating multipoles, we weight each $\mu$ bin by the normalized number of modes $N(k, \mu)$ counted on a $k$-space grid that is same as the grid used to estimate the measured power spectrum. More details of the correction method are given in B16. This effect, being apparent only at small $k$, does not influence the result of our analysis.

\subsection{Fitting preparation}

Using the covariance matrix we perform a $\chi^{2}$ minimization to find the best-fitting parameters. In addition to the scaling of the inverse covariance matrix of equation (26), we must propagate the error in the covariance matrix to the error on the estimated parameters; this is done by scaling the variance for each parameter by (Percival et al. 2014)

$M_{1}=\sqrt{\frac{1+B\left(n_{\mathrm{b}}-n_{\mathrm{p}}\right)}{1+A+B\left(n_{\mathrm{p}}+1\right)}}$, 
where $n_{\mathrm{p}}$ is the number of parameters and

$$
\begin{aligned}
A & =\frac{2}{\left(N_{\mathrm{s}}-n_{\mathrm{b}}-1\right)\left(N_{\mathrm{s}}-n_{\mathrm{b}}-4\right)}, \\
B & =\frac{N_{\mathrm{s}}-n_{\mathrm{b}}-2}{\left(N_{\mathrm{s}}-n_{\mathrm{b}}-1\right)\left(N_{\mathrm{s}}-n_{\mathrm{b}}-4\right)} .
\end{aligned}
$$

Using our post-reconstruction values of $N_{\mathrm{s}}=999$ for SGC and 996 for the NGC, $n_{\mathrm{b}}=58$ and $n_{\mathrm{p}}=16$, we obtain a correction of $M_{1} \approx 1.013$. When dealing with the variance or standard deviation of a distribution of finite mock results, which has also been fitted with a covariance matrix derived from the same mock results, the standard deviation from these mocks needs to be corrected as

$M_{2}=M_{1} \sqrt{\frac{N_{\mathrm{s}}-1}{N_{\mathrm{s}}-n_{\mathrm{b}}-2}}$.

\section{TESTING THE MODEL}

\subsection{Theoretical systematics}

While perturbation theory can attempt to provide a model for the non-linear power spectrum on quasi-linear scales $\left(k \leq 0.2 h^{-1} \mathrm{Mpc}\right)$, most observed modes are outside the realm of perturbation theory and it has proven very difficult to extract information from these modes. When focusing on the BAO feature, however, the two main non-linear effects are non-linear damping and an additional smallscale power due to mode coupling (Crocce \& Scoccimarro 2006; Eisenstein et al. 2007a; Matsubara 2008a; Seo et al. 2008, 2010):

$P_{\mathrm{g}}(k, \mu)=G^{2}(k, \mu, z) P_{\text {lin }}(k, \mu)+P_{\mathrm{MC}}$.

Here the propagator $G$ describes the cross-correlation between the initial and final density field, which is responsible for the damping of the BAO. In the high- $k$ limit, the dominant behaviour of the propagator can be predicted using perturbation theory (e.g. Crocce \& Scoccimarro 2006; Matsubara 2008a) as

$G \sim \exp \left(-\frac{1}{2} k^{2} \Sigma^{2}\right)$.

$\mathrm{N}$-body simulations have demonstrated that this form is a good approximation over the wave modes that are relevant to the BAO feature before reconstruction, and often even after reconstruction (e.g. Seo et al. 2010, 201). ${ }^{2}$

The mode coupling term in equation (58) can be written in the standard perturbation theory (PT; Jain \& Bertschinger 1994) as

$P_{\mathrm{MC}}(k) \simeq 2 \int\left[F_{2}(\boldsymbol{k}-\boldsymbol{q}, \boldsymbol{q})\right]^{2} P_{\mathrm{lin}}(\boldsymbol{q}) P_{\mathrm{lin}}(|\boldsymbol{k}-\boldsymbol{q}|) \mathrm{d} \boldsymbol{q}+\cdots$

with the second-order PT kernel

$F_{2}\left(\boldsymbol{k}_{1}, \boldsymbol{k}_{2}\right)=\frac{5}{7}+\frac{2}{7}\left(\frac{\boldsymbol{k}_{1} \cdot \boldsymbol{k}_{2}}{k_{1} k_{2}}\right)^{2}+\frac{\boldsymbol{k}_{1} \cdot \boldsymbol{k}_{2}}{2}\left(\frac{1}{k_{1}^{2}}+\frac{1}{k_{2}^{2}}\right)$.

The $F_{2}$-kernel divides the mode coupling term into three parts, where the first describes the growth of perturbations, the second represents the transport of matter by the velocity field and the last term describes the impact of tidal gravitational fields in the growth of structure. The leading contribution to the shift in the BAO scale results from the product of the first and second term (Crocce \& Scoccimarro 2006; Sherwin \& Zaldarriaga 2012). The amplitude of

\footnotetext{
${ }^{2}$ White (2015) and Seo et al. (2016) show that it depends on the convention and the details used in the reconstruction procedure.
}

this shift depends on redshift (through the growth factor) and galaxy bias (Seo et al. 2008, 2010; Padmanabhan \& White 2009; Mehta et al. 2011) and is approximately described by

$\alpha-1 \approx 0.5$ per cent $\left(1+\frac{3 b_{2}}{2 b_{1}}\right)[D(z) / D(0)]^{2}$,

where $D$ is the growth factor. Using the effective redshifts $z_{\text {eff }}=0.38$, 0.51 and 0.61 and assuming $b_{1}=2, b_{2}=0.2$ and $\Omega_{\mathrm{m}}=0.3$ within a flat $\Lambda \mathrm{CDM}$ cosmology, the equation above predicts systematic shifts of $\Delta \alpha=0.39,0.36$ and 0.33 per cent, respectively, without accounting for redshift-space distortions. These values are more than a factor of 2 times smaller than our best measurement uncertainties.

Furthermore, the technique of density field reconstruction, which we described in Section 6, has been shown to substantially undo the non-linear damping as well as remove the mode coupling bias (Seo et al. 2008; Padmanabhan \& White 2009; Sherwin \& Zaldarriaga 2012). While the efficiency of density field reconstruction depends on the noise level of the galaxy density field as well as various details used during reconstruction, Seo et al. (2008) demonstrated that the shifts are reduced to less than 0.1 percent even in the presence of non-negligible shot noise, implying the mode coupling term is quite robustly removed. In our analysis, the BAO constraints clearly improve after reconstruction to the degree that is consistent with the effect seen in the mock catalogues. This result suggests that reconstruction is working and therefore the mode coupling term should be removed. We therefore proceed without any treatment for a potential systematic bias due to mode coupling.

It has been suggested that the supersonic streaming velocity of baryons relative to dark matter at high redshift may have left an imprint in the low-redshift galaxy distribution such that the BAO scale shrinks or stretches relative to the conventional, zero-streaming velocity prediction (e.g. Dalal, Pen-L. \& Seljak 2010; Tseliakhovich \& Hirata 2010; Yoo, Dalal \& Seljak 2011; Beutler et al. 2016). Blazek, McEwen \& Hirata (2016) predict that a level of 1 per cent effect on the density fluctuation (i.e. streaming velocity bias) will induce $\mathrm{a} \sim 0.5$ per cent shift in the BAO scale. With little information on the magnitude and sign of the streaming velocity bias and its effect on the reconstruction process, we ignore a possible systematic bias due to this effect.

\subsection{Tests on $N$-body simulations}

To test our fitting technique we use two different sets of $\mathrm{N}$ body simulations, designated as runA and runPB. The runA simulations are 20 halo catalogues of size $\left[1500 h^{-1} \mathrm{Mpc}\right]^{3}$ with $1500^{3}$ particles using the fiducial cosmology of $\Omega_{\mathrm{m}}=0.274$, $\Omega_{\Lambda}=0.726, n_{\mathrm{s}}=0.95, \Omega_{\mathrm{b}}=0.0457, H_{0}=70 \mathrm{~km} \mathrm{~s}^{-1} \mathrm{Mpc}^{-1}$ and $r_{\mathrm{s}}\left(z_{\mathrm{d}}\right)=104.503 \mathrm{~h}^{-1} \mathrm{Mpc}$. The runPB simulations are 10 galaxy catalogues of size $\left[1380 h^{-1} \mathrm{Mpc}\right]^{3}$ with $\Omega_{\mathrm{m}}=0.292$, $\Omega_{\Lambda}=0.708, n_{\mathrm{s}}=0.965, \Omega_{\mathrm{b}}=0.0462, H_{0}=69 \mathrm{~km} \mathrm{~s}^{-1} \mathrm{Mpc}^{-1}$ and $r_{\mathrm{s}}\left(z_{\mathrm{d}}\right)=102.3477 h^{-1} \mathrm{Mpc}$. The runPB simulations use a CMASSlike halo occupation distribution (HOD) model to populate dark matter haloes with galaxies (see Reid et al. 2014 for details).

We calculate the power spectra for the runA and runPB simulations and fit the individual power spectra using the model described in Section 7. The results are summarized in Table 1 and displayed in Fig. 8.

In the case of the runA simulations, the pre-reconstruction results indicate a $2 \sigma$ bias towards larger values of $\alpha_{\|}$. This bias is not statistically significant but might be related to the mode coupling shift as discussed in Section 8.1. Our power spectrum model does not 
Table 1. Results for the fit to the mean of the runA and runPB simulations (in periodic boxes). The upper section of the table presents the fitting result using the power spectrum template of the correct cosmology, so that the different scaling parameters $\alpha$ should agree with unity and $\epsilon$ should agree with zero. The lower section of the table uses the runPB cosmology to define the power spectrum template when fitting the runA simulations and the runA cosmology for the fit to runPB simulations. Using a power spectrum template with a different sound horizon results in a shift of the different $\alpha$ s, given by the ratio of the true sound horizon to the used sound horizon. The rows with the label 'scaled' account for the difference in the sound horizon of the two templates, so that these values again should agree with unity. This represents a test of the scaling formalism used to retrieve the BAO scale. The sound horizon for runA is $104.503 h^{-1} \mathrm{Mpc}$ $(149.29 \mathrm{Mpc})$, while for runPB it is $102.3477 h^{-1} \mathrm{Mpc}(148.33 \mathrm{Mpc})$. The parameters $\alpha$ and $\epsilon$ are derived from $\alpha_{\|}$and $\alpha_{\perp}$. The fact that some values of $\alpha$ before reconstruction are larger than unity is consistent with the mode coupling term, which predicts a sub-per cent level shift to larger $\alpha$ (see Section 8.1). Mode coupling is removed after reconstruction.

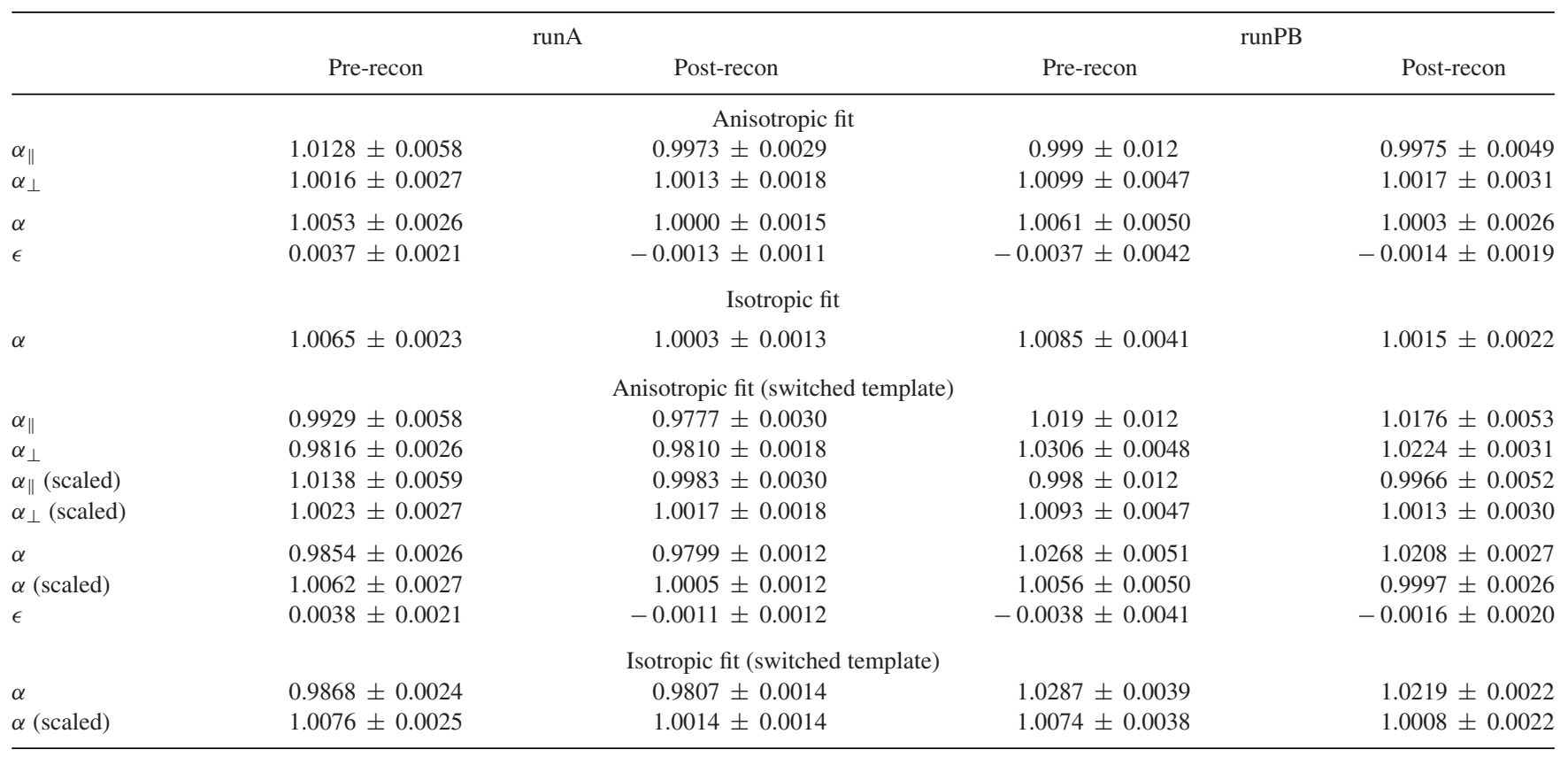
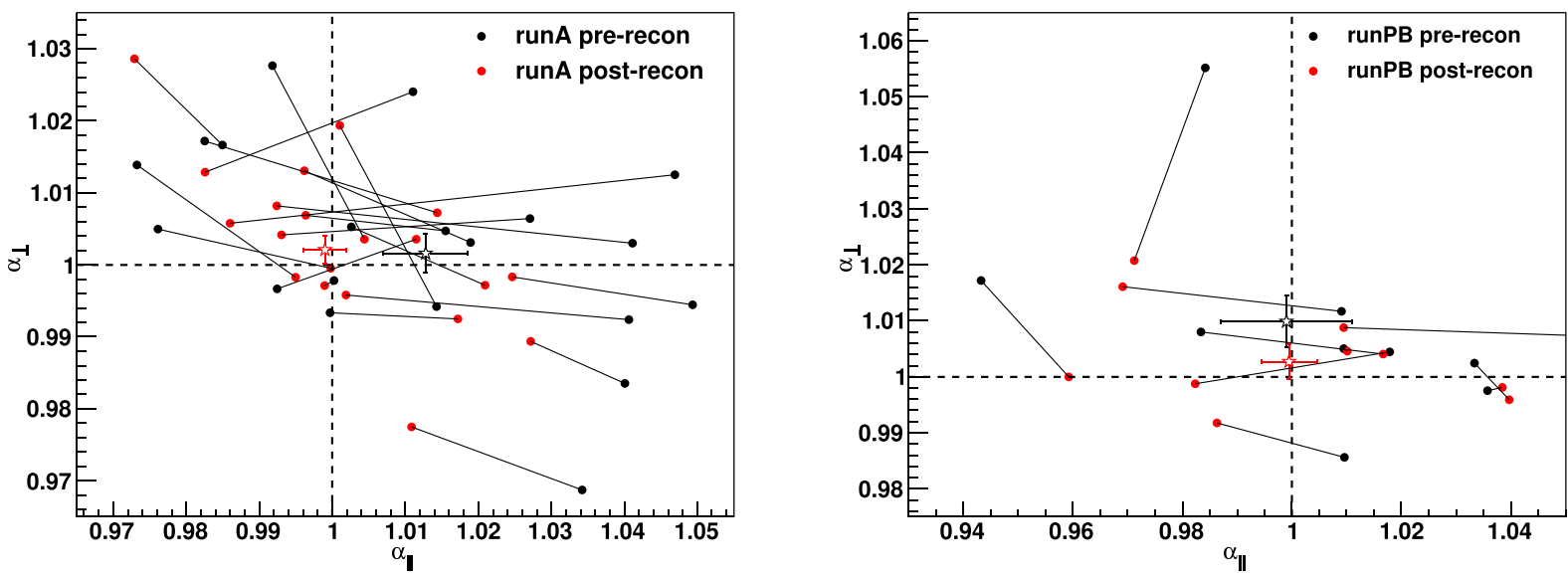

Figure 8. The distribution of $\alpha_{\|}$and $\alpha_{\perp}$ for the 20 realizations of the runA simulation (left) and the 10 realizations of the runPB simulation (right) before density field reconstruction (black) and after reconstruction (red). The star data points with error bars show the results of the fit to the mean of the simulation boxes again before (black) and after (red) reconstruction (the individual realizations are connected by black lines). The fact that the results before reconstruction (black) are biased to $\alpha>1$ is consistent with the mode coupling term. Mode coupling is removed after reconstruction. One of the 10 runPB realizations has $\alpha_{\|}=1.28$ before reconstruction, which indicates that for this realization there is no BAO detection along the line of sight.

account for the mode coupling term and therefore the presence of bias is expected. However, the mode coupling term should be removed after applying density field reconstruction. Our postreconstruction results are indeed consistent with $\alpha=1$, indicating no systematic bias in our measurements.

The results for the runPB simulations (Fig. 8, right) are quite similar, even though instead of having a $2 \sigma$ bias in $\alpha_{\|}$pre-reconstruction we now find a $2 \sigma$ bias in $\alpha_{\perp}$. Again our post-reconstruction results are unbiased.

We also performed tests where we switched the input power spectrum model using the runPB cosmology for the fit to runA and the other way around. These results are included in Table 1 with the label 'switched template'. For these fits an unbiased result does not mean agreement with $\alpha=1$, since the cosmology assumed in 

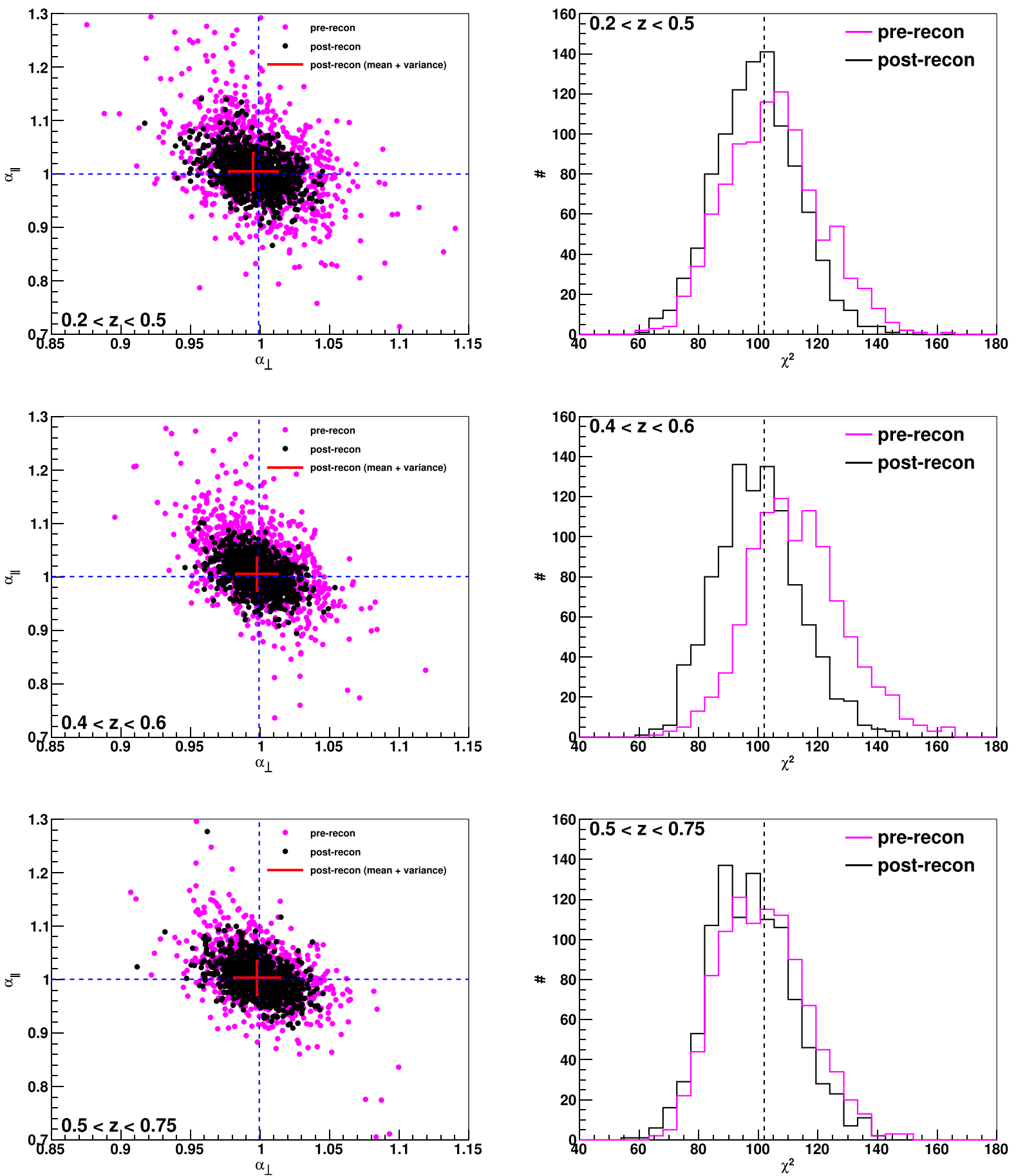

Figure 9. Maximum likelihood values for the MultiDark-Patchy mock catalogues (left) and the corresponding minimum, $\chi^{2}$ distribution (right) for the three redshift bins used in this analysis. The magenta data points on the left and the magenta solid line on the right show the pre-reconstruction results, while the black points (left) and the black line (right) present the post-reconstruction results. The red crosses in the left-hand panels are the mean and variance for the mock catalogues post-reconstruction. The black dashed line on the right indicates the degrees of freedom. The results are summarized in Table 2.

the model is different from the true cosmology of the simulation. The results with the label 'scaled' show the fitting results that are adjusted with the ratio between the cosmology in the template and the input cosmology of the simulations, where now these results can be compared to unity. We found the results are consistent with our previous findings, i.e. no bias on the measured BAO scale.

\subsection{Tests on the MultiDark-Patchy mock catalogues}

The tests in the last section have been performed on simulations with periodic boundary conditions, which do not take into account the survey geometry of BOSS. Here we use the MultiDark-Patchy mock catalogues, introduced in Section 5, which incorporate the BOSS survey geometry. The mean of the MultiDark-Patchy power 
Table 2. The results for the fits to the MultiDark-Patchy mock catalogues for the three redshift bins used in this analysis before and after density field reconstruction. For each bin we present the anisotropic results $\left(\alpha_{\|}, \alpha_{\perp}\right)$ and the isotropic result $(\alpha)$. The anisotropic results also show the correlation coefficient $r$ between the two $\alpha$-parameters. The fiducial BOSS cosmology is used when analysing the mock data, which means that the $\alpha$ values do not have to agree with unity. The expectation value for each redshift bin is given in brackets. The uncertainties represent the variance between all mock catalogues (not the error on the mean).

\begin{tabular}{|c|c|c|c|c|}
\hline & Pre-recon & $r_{\text {pre-recon }}$ & Post-recon & $r_{\text {post-recon }}$ \\
\hline & \multicolumn{4}{|c|}{$0.2<z<0.5$} \\
\hline$\alpha_{\|}$ & $1.018 \pm 0.076(0.9999)$ & -0.455 & $1.005 \pm 0.036(0.9999)$ & -0.398 \\
\hline$\alpha_{\perp}$ & $0.999 \pm 0.031(0.9991)$ & & $0.995 \pm 0.018(0.9991)$ & \\
\hline \multirow[t]{2}{*}{$\alpha$} & $1.010 \pm 0.022(0.9993)$ & - & $1.002 \pm 0.013(0.9993)$ & - \\
\hline & \multicolumn{4}{|c|}{$0.4<z<0.6$} \\
\hline$\alpha_{\|}$ & $1.019 \pm 0.066$ & -0.482 & $1.005 \pm 0.032$ & -0.397 \\
\hline$\alpha_{\perp}$ & $0.999 \pm 0.027(0.9993)$ & & $0.998 \pm 0.016(0.9993)$ & \\
\hline \multirow[t]{2}{*}{$\alpha$} & $1.010 \pm 0.019(0.9996)$ & - & $1.003 \pm 0.012(0.9996)$ & - \\
\hline & \multicolumn{4}{|c|}{$0.5<z<0.75$} \\
\hline$\alpha_{\|}$ & $1.010 \pm 0.053(1.0006)$ & -0.464 & $1.003 \pm 0.033$ & -0.413 \\
\hline$\alpha_{\perp}$ & $1.000 \pm 0.024(0.9995)$ & & $0.998 \pm 0.017(0.9995)$ & \\
\hline$\alpha$ & $1.008 \pm 0.019(0.9999)$ & - & $1.003 \pm 0.012(0.9999)$ & - \\
\hline
\end{tabular}

Table 3. The constraints from the BOSS DR12 data analysis, representing the main results of this paper. The upper section presents the anisotropic fits, while the lower part lists the isotropic results. The fitting range is $k=0.01-0.3 \mathrm{~h} \mathrm{Mpc}^{-1}$ for both monopole and quadrupole. We include the result before (pre-recon) and after (post-recon) density field reconstruction. The $\alpha$ and $\epsilon$ values are derived from the $\alpha_{\|}$and $\alpha_{\perp}$ values using equation (43). We also show the constraints on the Alcock-Paczynski parameter $F_{\mathrm{AP}}(z)=(1+z) D_{\mathrm{A}}(z) H(z) / c$ and the isotropic distance scale $D_{V}(z)=\left[(1+z)^{2} D_{\mathrm{A}}^{2}(z) c z / H(z)\right]^{1 / 3}$. The uncertainties are derived from the 68 per cent confidence levels. The results are displayed in Figs 12-14. The correlation between $\alpha_{\perp}$ and $\alpha_{\|}$as derived from the measurement likelihood is $-0.378,-0.389$ and -0.464 for the low-, middle- and high-redshift bins, respectively.

\begin{tabular}{|c|c|c|c|c|c|c|c|}
\hline & & \multicolumn{2}{|c|}{$0.2<z<0.5$} & \multicolumn{2}{|c|}{$0.4<z<0.6$} & \multicolumn{2}{|c|}{$0.5<z<0.75$} \\
\hline & & Pre-recon & Post-recon & Pre-recon & Post-recon & Pre-recon & Post-recon \\
\hline \multicolumn{8}{|c|}{ Anisotropic fit } \\
\hline$\alpha_{\perp}$ & & $0.981 \pm 0.021$ & $0.984 \pm 0.016$ & $1.008 \pm 0.023$ & $0.997 \pm 0.013$ & $1.009 \pm 0.025$ & $1.000 \pm 0.015$ \\
\hline$\chi^{2} /$ d.o.f. & & $109.9 /(116-16)$ & $101.2 /(116-16)$ & $105.6 /(116-16)$ & $68.0 /(116-16)$ & $96.8 /(116-16)$ & $97.2 /(116-16)$ \\
\hline$\alpha$ & & $1.002 \pm 0.015$ & $0.999 \pm 0.011$ & $1.009 \pm 0.016$ & $0.993 \pm 0.0091$ & $0.987 \pm 0.017$ & $0.988 \pm 0.0090$ \\
\hline$D_{V}(z) r_{\mathrm{s}}^{\mathrm{fid}} / r_{\mathrm{s}}$ & $(\mathrm{Mpc})$ & $1479 \pm 23$ & $1474 \pm 17$ & $1903 \pm 30$ & $1873 \pm 17$ & $2141 \pm 36$ & $2144 \pm 20$ \\
\hline$H(z) r_{\mathrm{s}} / r_{\mathrm{s}}^{\text {fid }}$ & $\left(\mathrm{km} \mathrm{s}^{-1} \mathrm{Mpc}^{-1}\right)$ & $79.3 \pm 2.8$ & $80.7 \pm 2.4$ & $88.7 \pm 4.3$ & $90.8 \pm 2.0$ & $101.1 \pm 4.4$ & $98.9 \pm 2.3$ \\
\hline$D_{\mathrm{A}}(z) r_{\mathrm{s}}^{\mathrm{fid}} / r_{\mathrm{s}}$ & $(\mathrm{Mpc})$ & $1088 \pm 23$ & $1092 \pm 18$ & $1323 \pm 30$ & $1308 \pm 18$ & $1446 \pm 36$ & $1433 \pm 21$ \\
\hline \multicolumn{8}{|c|}{ Isotropic fit } \\
\hline$\alpha$ & & $1.006 \pm 0.016$ & $1.000 \pm 0.010$ & $1.016 \pm 0.017$ & $0.9936 \pm 0.0082$ & $0.991 \pm 0.019$ & $0.9887 \pm 0.0087$ \\
\hline$D_{V}(z) r_{\mathrm{s}}^{\mathrm{fid}} / r_{\mathrm{s}}$ & (Mpc) & $1485 \pm 24$ & $1476 \pm 15$ & $1916 \pm 32$ & $1874 \pm 16$ & $2150 \pm 42$ & $2146 \pm 19$ \\
\hline
\end{tabular}

spectra (we have 2045 pre-reconstruction power spectra and 996 post-reconstruction power spectra for the NGC and 2048 prereconstruction and 999 post-reconstruction power spectra for the $\mathrm{SGC}$ ) is included in Figs 1 and 2 for comparison with the data measurements.

We fitted each individual mock catalogue and included the maximum likelihood value in Fig. 9 (left). The black data points correspond to the post-reconstruction results, while the magenta points show the pre-reconstruction results. The results are also included in Table 2, where we list the mean and variance between the mock results. The largest offset between our mean post-recon results and the true underlying cosmology is 0.5 per cent, less than $1 / 6$ of the standard deviation expected in these measurements.

The distribution of maximum likelihood results for the MultiDark-Patchy mock catalogues indicates a correlation between $\alpha_{\perp}\left(\propto D_{\mathrm{A}}\right)$ and $\alpha_{\|}\left(\propto H^{-1}\right)$ of $\sim-0.47$ pre-reconstruction, while this value increases to $\sim-0.4$ in our post-reconstruction results.
Fisher matrix forecasts predict a correlation value of $\approx-0.41$ (i.e. anticorrelated $D_{\mathrm{A}}$ and $H^{-1}$ ) pre- and post-reconstruction (Seo \& Eisenstein 2003, 2007) for the BAO-only analysis, i.e. when we marginalize over any redshift-space distortion effects. Our postreconstruction values are in good agreement with the Fisher matrix predictions, while for pre-reconstruction the correlation is smaller (i.e. more negative) than expected. In the limit of the pure AlcockPaczynski test, i.e. when we have a constraint only on $D_{\mathrm{A}} H$, we expect a correlation of unity between $\alpha_{\perp}$ and $\alpha_{\|}$; using less information from the BAO scale will therefore increase the contribution from the Alcock-Paczynski test and push the correlation towards 1 from -0.4 (Seo \& Eisenstein 2003; Shoji, Jeong, \& Komatsu 2009). The pre-reconstruction correlation coefficient we observe is therefore not easily explained even if we assume a potential inclusion of non-BAO information. Note, however, that the Fisher matrix forecast assumes complete information of $P(k, \mu)$, while our data include only the monopole and quadrupole (excluding the 


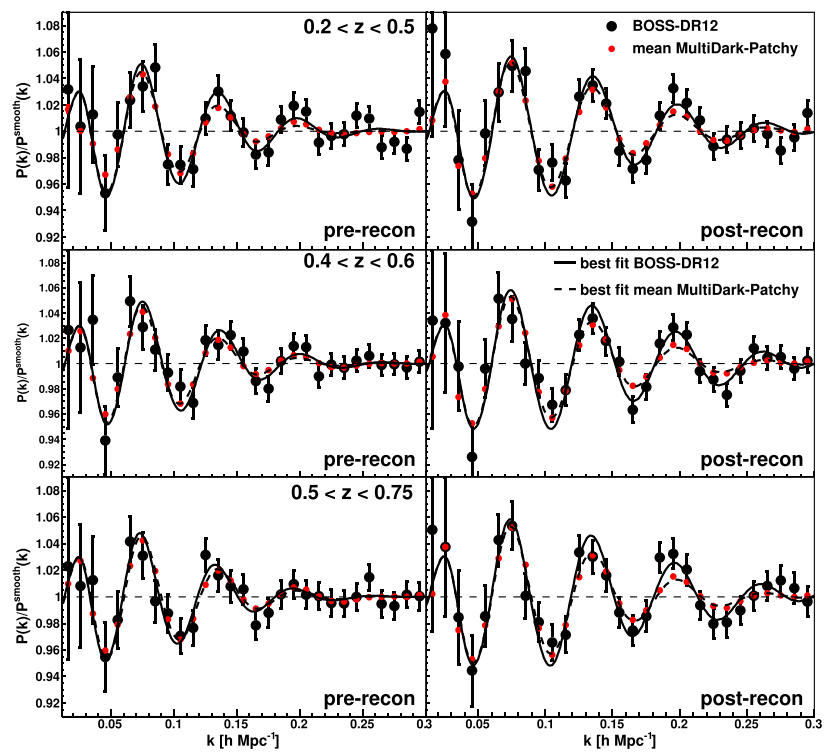

Figure 10. The monopole power spectrum measurements (black data points) relative to the best-fitting smooth power spectrum of equation (50). The left-hand panel shows the pre-reconstruction results and the right-hand panel presents the post-reconstruction results. The black solid line represents the best-fitting model to the data. The red data points are the mean power spectrum monopole of the MultiDark-Patchy mock catalogues and the black dashed line shows the best fit to the red data points. We observe a larger damping of the BAO signal in the MultiDark-Patchy mock catalogues compared to the data (see discussion Section 8.3 for details).

hexadecapole). The configuration space analysis of Ross et al. (2016) found $r \sim-0.49$ pre-reconstruction and $r \sim-0.4$ postreconstruction. These values agree well with our findings. The consensus result of the BOSS DR11 analysis (Anderson et al. 2014) had $r=-0.54$ post-reconstruction, significantly more negative than our DR12 correlation as well as the Fisher prediction. ${ }^{3}$

The rms between the maximum likelihood results of the MultiDark-Patchy mock catalogues is larger than the constraints we observe in the BOSS-DR12 data catalogues by $\sim 30$ percent (compare Table 2 with Table 3). To understand this discrepancy, we now investigate how well the MultiDark-Patchy mock catalogues represent the data in terms of the BAO signal. Fig. 10 presents the isotropic BAO signal in the data (black data points) compared to the mean of the MultiDark-Patchy mock catalogues (red data points). The mock catalogues show a larger damping of the BAO signal compared to the data, which is more prominent post-reconstruction.

Post-reconstruction only 1.9 per cent of the mock catalogues in the high-redshift bin and 7.7 per cent of the mocks in the low-redshift bin have smaller uncertainties than the data. To investigate this tension we first look at the measured damping scale in the data, where for simplicity we focus on the isotropic case. The best-fitting damping scales pre-reconstruction are $\Sigma_{\mathrm{NL}}=8.3 \pm 1.4,8.8_{-1.3}^{+1.6}$ and $9.8_{-1.6}^{+2.1} h^{-1} \mathrm{Mpc}$ for the low-, middle- and high-redshift bins, respectively. After density field reconstruction, we get $\Sigma_{\mathrm{NL}}=5.0 \pm$ $1.2,3.4_{-3.0}^{+1.4}$ and $3.2_{-4.3}^{+1.6} h^{-1} \mathrm{Mpc}$. We can compare these measurements with the expectations given by the Zel'dovich approximation

\footnotetext{
${ }^{3}$ The DR11 analysis used $R=1$ in equation (35) for the post-reconstruction power spectrum model. We find this old fitting model indeed tends to lead to more negative correlation.
}

(e.g. Matsubara 2008a):

$$
\begin{aligned}
& \Sigma_{x y}^{2}=\frac{1}{3 \pi^{2}} \int \mathrm{d} p P_{\operatorname{lin}}(p, z), \\
& \Sigma_{z}=(1+f) \Sigma_{x y} .
\end{aligned}
$$

We approximate the damping of the spherically averaged power spectrum to be

$\Sigma_{\mathrm{NL}}=\sqrt{\frac{2}{3} \Sigma_{x y}^{2}+\frac{1}{3} \Sigma_{z}^{2}}$.

This predicts $\Sigma_{\mathrm{NL}}=8.8 h^{-1} \mathrm{Mpc}$ at $z=0.38, \Sigma_{\mathrm{NL}}=8.4 h^{-1} \mathrm{Mpc}$ at $z=0.51$ and $\Sigma_{\mathrm{NL}}=8.1 h^{-1} \mathrm{Mpc}$ at $z=0.61$ before reconstruction. These values are slightly smaller than our measurements but consistent within the measurement uncertainties. The expected damping scale post-reconstruction does depend on the effectiveness of reconstruction, which depends on e.g. survey geometry. Seo et al. (2016) measure $\Sigma_{\mathrm{NL}} \sim 4.3 h^{-1} \mathrm{Mpc}$ at redshift $z \sim 0.57$ postreconstructing, which agrees with our measurements. The PTHalo mock catalogues (Manera et al. 2013, 2015) used in the BOSS DR9 (Anderson et al. 2012) and DR10/11 (Anderson et al. 2014) analysis showed a damping of $\Sigma_{\mathrm{NL}} \sim 4.6$ and $\sim 4.8 h^{-1} \mathrm{Mpc}$ at redshift 0.57 and 0.32 , respectively, again agreeing with out measurements. Meanwhile, the MultiDark-Patchy mocks show $\Sigma_{\mathrm{NL}} \sim 7 h^{-1} \mathrm{Mpc}$ post-reconstruction. We therefore conclude that the BAO signal from the BOSS DR12 data set is consistent with the expectation, while the MultiDark Patchy mocks tend to underestimate the BAO signal. We believe this excess damping is a limitation of the 2LPT approximation used in the mock production (for more details about the MultiDark-Patchy mock production see Kitaura et al. 2016). The effect is presumably more apparent post-reconstruction because it is hidden by the larger intrinsic damping pre-reconstruction.

The larger damping scale in the MultiDark-Patchy catalogues is clearly visible when fitting the mock power spectra and comparing to the BOSS-DR12 results (see Fig. 11). Given that the main purpose of the mock catalogues is to estimate the band power precision of our power spectrum measurements, these effects do not impact our analysis. However, one should keep these effects in mind when using these catalogues to study the BAO signal. We therefore consider our pipeline tests with the $N$-body simulations as more robust.

\section{DR12 DATA ANALYSIS}

\subsection{The anisotropic fit}

Fig. 12 compares the best-fitting power spectrum multipole models with the measurements. The best fit for the SGC (red solid line) and the NGC (black solid line) use different amplitude parameters $B$ to account for potential differences due to target selection. Except for the amplitude all parameters are identical and we obtain these results by fitting the NGC and SGC simultaneously. The lower two panels in Fig. 12 show the residuals for the monopole and quadrupole separately, indicating a good fit on all scales. The best-fitting $\chi^{2} /$ d.o.f. is $101.2 /(116-16), 68.0 /(116-16)$ and $97.2 /(116-16)$. The probabilities of having reduced $\chi^{2}$ values that exceed these values are $44.8,99.4$ and 56.1 per cent. The middle-redshift bin has a $\chi^{2}$ below expectation (with a significance $<3 \sigma$ ), which might be related to the higher amplitude of the MultiDark-Patchy mocks for this redshift bin that overestimates the uncertainties (see Fig. 1).

The correlation between $\alpha_{\perp}$ and $\alpha_{\|}$as derived from the measurement likelihood is $-0.378,-0.389$ and -0.464 for the low-, middleand high-redshift bins, respectively. These values are very similar 

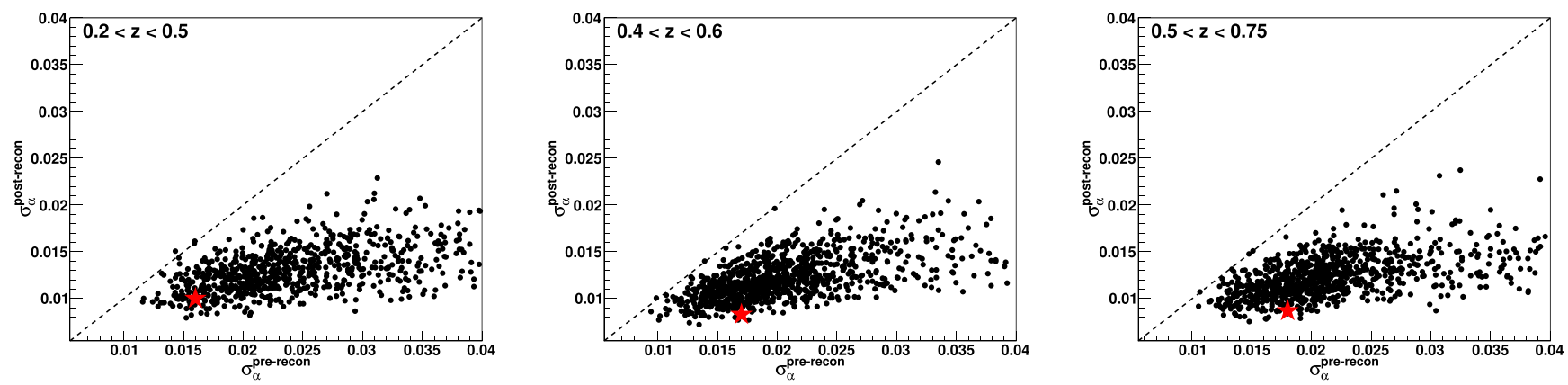

Figure 11. The uncertainties on the angular average distance scale parameter $\alpha$ before and after density field reconstruction. The red stars show the measurement in BOSS-DR12, while the black points indicate the results for the MultiDark-Patchy mock catalogues.
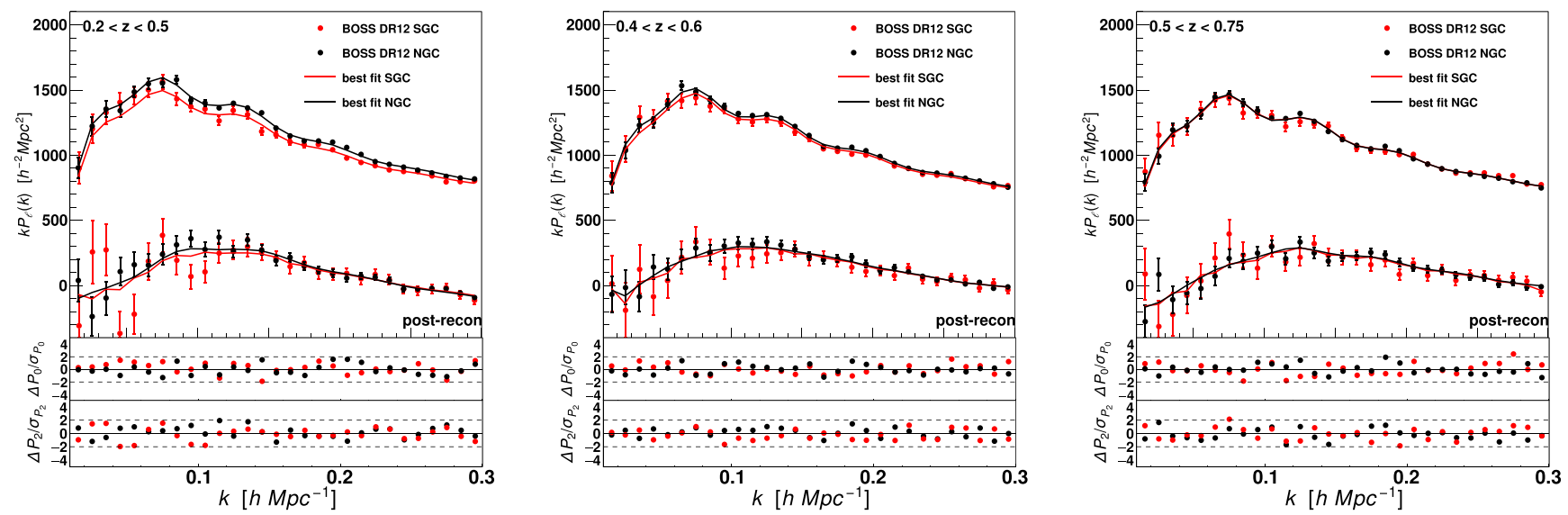

Figure 12. Comparison between the best-fitting model and the BOSS DR 12 measurements in the three redshift bins used in this analysis. The errors on the data points are the diagonal of the corresponding covariance matrix. The red line represents the best-fitting model to the SGC, while the black line shows the best-fitting model for the NGC. The SGC best-fitting model includes a small discreteness effect mainly visible at small $k$. The NGC and SGC have been fit simultaneously, using the same cosmological fitting parameters. However, the SGC and NGC have a separate amplitude nuisance parameter and different window functions, which leads to the difference between the red and black line. The reason for having separate nuisance parameters for NGC and SGC is slight differences in the galaxy sample selection (see Section 2 and Alam et al. 2016). See Table 3 for more details.

to $-0.398,-0.397$ and -0.413 , which are the corresponding values from the Multidark-Patchy mock catalogues.

The best-fitting BAO scale parameters for the three redshift bins are shown in Table 3. For all redshift bins we found significant (up to a factor of 2) improvements after applying density field reconstruction. Post-reconstruction we have 2.9, 2.2 and 2.3 per cent constraints on $\alpha_{\|}$for the low- $\left(z_{\text {eff }}=0.38\right)$, middle- $\left(z_{\text {eff }}=0.51\right)$ and high-redshift bin $\left(z_{\text {eff }}=0.61\right)$, respectively. For $\alpha_{\perp}$, the postreconstruction constraints are $1.6,1.3$ and 1.5 per cent at $z_{\text {eff }}=0.38$, 0.51 and 0.61 , respectively.

\subsection{The isotropic fit}

The best-fitting results in the case of the isotropic analysis (monopole only) are shown in Fig. 14. The best post-reconstruction constraints are $\alpha=1.000 \pm 0.010,0.9936 \pm 0.0082$ and 0.9887 \pm 0.0087 at $z_{\text {eff }}=0.38,0.51$ and 0.61 , respectively. Thus we have two independent (the middle-redshift bin is correlated with the other two) $\sim 1$ percent distance constraints at the low- and at high-redshift bins: 0.88 percent at $z=0.61$ and 1 percent at $z_{\text {eff }}=0.38$. The best-fitting reduced $\chi^{2}$ in the isotropic case is $\chi^{2} /$ d.o.f. is $48.5 /(58-10), 64.8 /(58-10)$ and $49.8 /(58-10)$ for pre-reconstruction and $43.9 /(58-10), 32.8 /(58-10)$ and $47.0 /(58-10)$ for post-reconstruction fits. The second redshift bin shows a large $\chi^{2}$ pre-reconstruction and a fairly small $\chi^{2}$ postreconstruction. The probability to have a reduced $\chi^{2}$ value that exceeds this value is 6.5 percent for the pre-reconstruction value and 96.4 per cent for the post-reconstruction result, meaning that these results are $2 \sigma$ fluctuations from expectation.

\section{DISCUSSION}

The likelihood distribution for our best-fitting isotropic and anisotropic results in terms of $D_{\mathrm{A}}(z)$ and $H(z)$ are displayed in Fig. 13, together with the likelihood distribution for Planck within $\Lambda \mathrm{CDM}$. Including the quadrupole our anisotropic analysis can break the degeneracy between $D_{\mathrm{A}}$ and $H$ and constrain both parameters separately. Our constraints for all redshift bins are in good agreement with the Planck prediction within $\Lambda \mathrm{CDM}$.

\subsection{Comparison to other DR12 BAO measurements}

Ross et al. (2016) analysed the same BOSS DR12 data as used in this analysis in configuration space, finding overall good agreement (any difference is $<0.5$ percent) for the best-fitting values and for the measurement uncertainties. We find a correlation of $\sim 0.9$ between our BAO constraints and the constraints of Ross et al. (2016). Our companion paper (Alam et al. 2016) combines the BAO constraint 

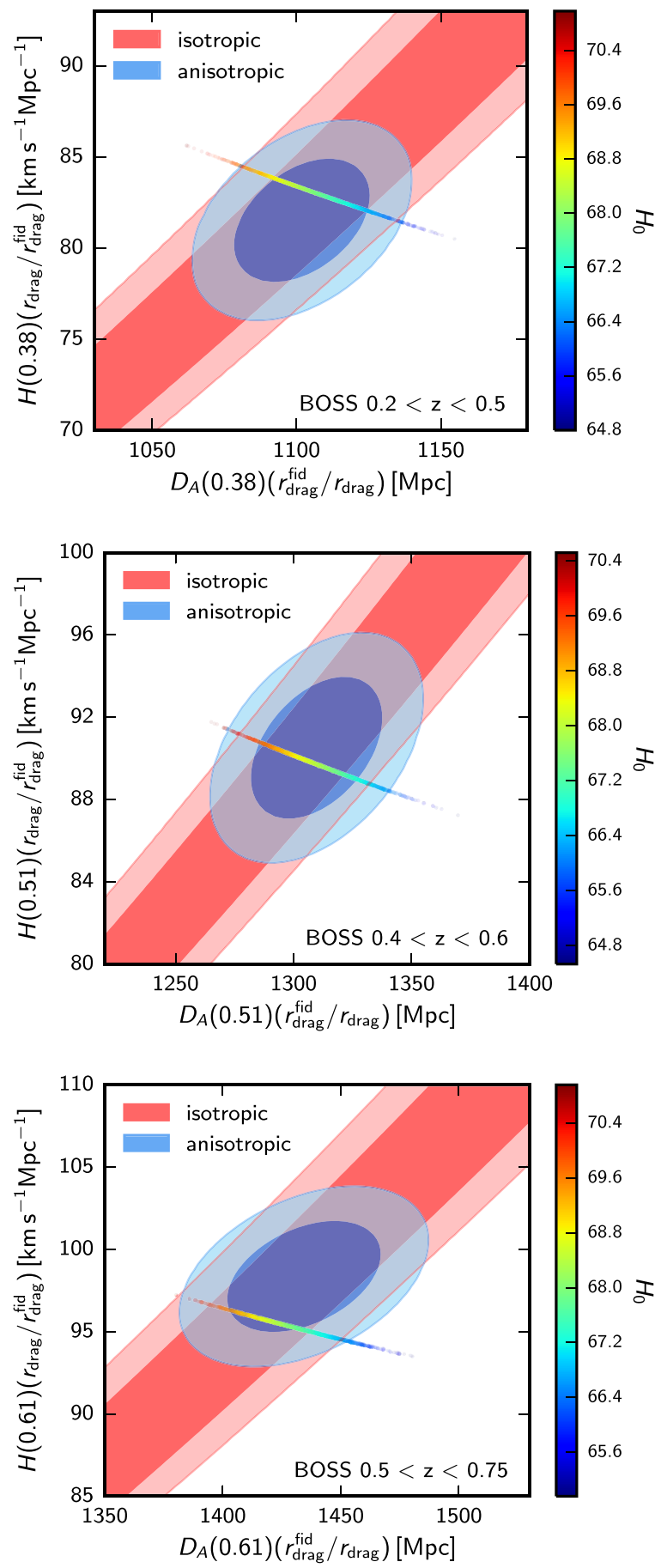

Figure 13. The likelihood distribution for the fit to the BOSS power spectrum multipoles for the three redshift bins. The red contours show the isotropic (monopole only) fit, while the blue contours present the anisotropic (monopole+quadrupole) fit. We added the likelihood distribution for $H_{0}$ measured by Planck (Planck2015+lensing) where we assumed a $\Lambda \mathrm{CDM}$ model to extrapolate from the redshift of decoupling to the effective redshifts of the three samples. See Table 3 for more details. derived in this paper with the results in Ross et al. (2016) to obtain a combined likelihood, representing the final BOSS BAO result.

In Gil-Marin et al. (2016) the BOSS DR12 sample has been analysed using the two redshift bins of CMASS and LOWZ. While the CMASS results agree quite well with our high-redshift bin, there are some differences between our low-redshift bin and the LOWZ result. The LOWZ sample is defined by the redshift range $0.15-0.43$, while our low-redshift sample covers $0.2<z<0.5$. The combined sample used in our analysis also includes some new data based on the 'early regions' (see Section 2), which have been excluded in LOWZ.

\subsection{Significance of the BAO detection}

We can test the significance of the detection of the BAO signal in BOSS by comparing our results to the limit $\Sigma_{\mathrm{NL}} \rightarrow \infty$, which corresponds to a power spectrum without the BAO signal. We focus here on the isotropic analysis. Before applying density field reconstruction, our no-BAO fits result in a $\chi^{2}$ of $60.2,82.1$ and 67.4 for the low-, middle- and high-redshift bin, respectively. Comparing to the best-fitting $\chi^{2}$ in Table 3 we have $\Delta \chi^{2}=11.7,17.3$ and 17.6, which indicate detection significances of $3.4 \sigma, 4.2 \sigma$ and $4.2 \sigma$. After applying density field reconstruction, the $\chi^{2}$ for the no-BAO fits is $106.2,97.3$ and 113.6 for the low-, middle- and high-redshift bins, respectively. Again comparing to Table 3 we have $\Delta \chi^{2}=62.3,64.5$ and 66.6, which indicate detection significances of $7.9 \sigma, 8.0 \sigma$ and $8.2 \sigma$.

\subsection{Comparison to the Fisher matrix forecasts}

As a check that our results are close to optimal, and to assess the reliability of predictions for the future, it is useful to compare our results to pre-survey predictions of BOSS's BAO measuring power (Eisenstein et al. 2011) based on the code of Seo \& Eisenstein (2007). The details take us too far afield so are given in Appendix C, with the following summary.

(i) Our measurements of $\alpha$, which is approximately equivalent to the dilation factor $R$ of Seo \& Eisenstein (2007), can be aggregated to an overall 0.70 per cent distance error.

(ii) Eisenstein et al. (2011) predicted an error 1.44 times smaller, 0.48 per cent.

(iii) Our measured BAO errors are typical given the measured band power covariance, i.e. there is no evidence that the measurement was unlucky in its error bars. The aggregated error averaged over many MultiDark-Patchy mock catalogues is 0.88 per cent, an even worse 1.82 times larger than the Fisher forecast. We believe this is because the BAO signal is smaller than it should be in these mocks (see Section 8.3), not that this is evidence that the measurement on the data was lucky.

(iv) We found that the measured galaxy bias is lower than what was used for the original Fisher matrix forecast, which has a significant effect on the expected BAO constraints. Using the measured number of galaxies and their measured bias predicts a $\mathrm{BAO}$ error of 0.68 per cent, very close to our measurement. The Fisher predictions for the measured band power errors, using the pre-survey expected number of galaxies and bias (instead of measured), predict an aggregated error of 0.59 per cent. Here we are using the Planck cosmology.

(v) The Fisher predictions for the measured band power errors, using the pre-survey expected number of galaxies and bias, and the 3-year Wilkinson Microwave Anisotropy Probe (WMAP3) 


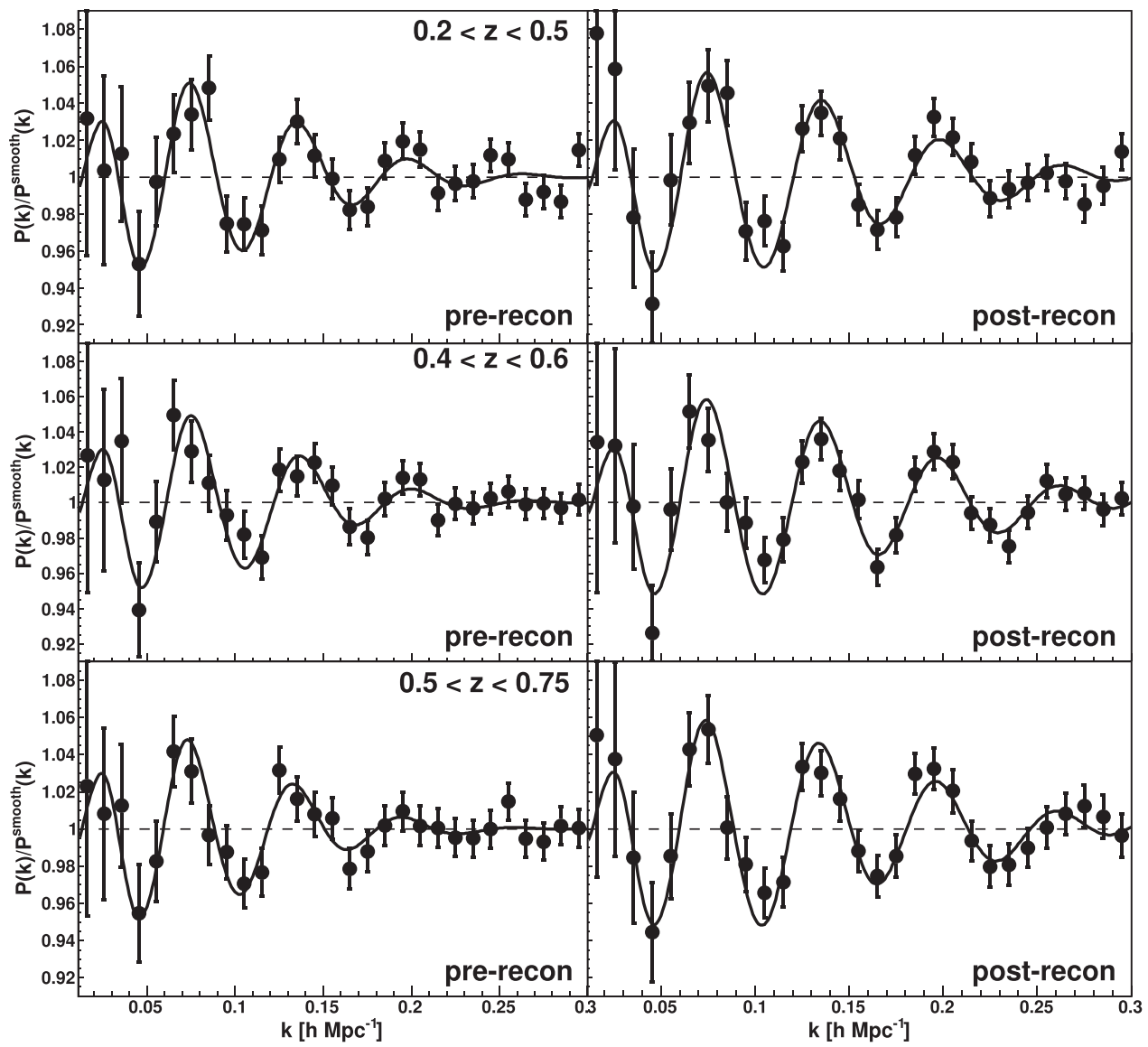

Figure 14. The best-fitting models (black solid line) of the isotropic BAO analysis compared to the power spectrum monopole measurements (data points). Both model and data have been plotted relative to the smooth model, and the data points for NGC and SGC have been combined using the corresponding covariance matrices (see Appendix B). The left-hand panel shows the pre-reconstruction result, while the right-hand panel presents the post-reconstruction result. Similar plots for the NGC and SGC separately are included in Appendix A. See Table 3 for more details.

cosmology, which is hard-coded into the Seo \& Eisenstein (2007) code, predict an aggregated error of 0.51 per cent, approximately equivalent to the 0.48 per cent expectation based on the Seo \& Eisenstein (2007).

Therefore we conclude that approximately half the difference between the pre-survey expectation and the achieved measurement $(\sim 0.59 / 0.51)$ is due to the Planck cosmology being less favourable for $\mathrm{BAO}$ than WMAP3 (e.g. lower baryon/CDM ratio) with another half $(\sim 0.68 / 0.59)$ being lower than expected bias. Note that contributing effects do not add linearly, and there are various smaller effects not mentioned in this summary. There is no sign of suboptimality in the data analysis.

\section{CONCLUSION}

We have measured the power spectrum multipoles from the final BOSS DR12 data set in three (overlapping) redshift bins, covering the total redshift range $0.2<z<0.75$. Our analysis focuses on measuring the isotropic and anisotropic BAO signal in the Fourier space. Our main results are the following.

(i) We measure the power spectrum monopole and quadrupole, accounting for the window function, aliasing and discreteness effects and extract the BAO information by marginalizing over the broad-band shape of the power spectrum. We validate our analy- sis pipeline using two sets of $N$-body simulations as well as the MultiDark-Patchy mock catalogues.

(ii) Fitting the monopole and quadrupole between $k=0.01$ and $0.30 h^{-1} \mathrm{Mpc}$ produces a constraint on the Hubble parameter of $H(z) r_{\mathrm{s}} / r_{\mathrm{s}}^{\text {fid }}=79.3 \pm 2.8 \mathrm{~km} \mathrm{~s}^{-1} \mathrm{Mpc}^{-1}$ and a constraint on the angular diameter distance of $D_{\mathrm{A}}(z) r_{\mathrm{s}}^{\mathrm{fid}}=1088 \pm 23 \mathrm{Mpc}$ for the low-redshift bin and $H(z) r_{\mathrm{s}} / r_{\mathrm{s}}^{\text {fid }}=98.9 \pm 2.3 \mathrm{~km} \mathrm{~s}^{-1} \mathrm{Mpc}^{-1}$ and $D_{\mathrm{A}}(z) r_{\mathrm{s}}^{\text {fid }}=1433 \pm 21 \mathrm{Mpc}$ for the high-redshift bin (see Table 3 for a complete summary of the results). While the high-redshift bin is in good agreement with previous results from the CMASS sample, our low-redshift constraint is significantly improved compared to previous studies. Our results are included in Alam et al. (2016), where a detailed study of the cosmological implications is performed.

(iii) Ignoring the Alcock-Paczynski effect we can constrain the angular averaged distance, $D_{V}$, for which we obtain a 1 per cent and a 0.88 per cent constraints at the effective redshifts of $z_{\text {eff }}=0.38$ and 0.61 , respectively.

(iv) The detection significances of the BAO signal are $3.4 \sigma, 4.2 \sigma$ and $4.2 \sigma$ before applying density field reconstruction for the low-, middle- and high-redshift bins, respectively, and increases to $7.9 \sigma$, $8.0 \sigma$ and $8.2 \sigma$ after density field reconstruction.

Alam et al. (2016) combine our measurements with the corresponding correlation function measurements of Ross et al. (2016) and the growth of structure measurements of Beutler et al. (2016), 
Grieb et al. (2016), Sanchez et al. (2016) and Satpathy et al. (2016) into a final BOSS likelihood and investigate the cosmological implications.

\section{ACKNOWLEDGEMENTS}

FB acknowledges support from the UK Space Agency through grant ST/N00180X/1.

Funding for SDSS-III has been provided by the Alfred P. Sloan Foundation, the Participating Institutions, the National Science Foundation and the US Department of Energy Office of Science. The SDSS-III web site is http://www.sdss3.org/.

SDSS-III is managed by the Astrophysical Research Consortium for the Participating Institutions of the SDSS-III Collaboration including the University of Arizona, the Brazilian Participation Group, Brookhaven National Laboratory, Carnegie Mellon University, University of Florida, the French Participation Group, the German Participation Group, Harvard University, the Instituto de Astrofisica de Canarias, the Michigan State/Notre Dame/JINA Participation Group, Johns Hopkins University, Lawrence Berkeley National Laboratory, Max Planck Institute for Astrophysics, Max Planck Institute for Extraterrestrial Physics, New Mexico State University, New York University, Ohio State University, Pennsylvania State University, University of Portsmouth, Princeton University, the Spanish Participation Group, University of Tokyo, University of Utah, Vanderbilt University, University of Virginia, University of Washington and Yale University.

This research used resources of the National Energy Research Scientific Computing Center, which is supported by the Office of Science of the US Department of Energy under Contract No. DEAC02-05CH11231.

H-JS is supported by the US Department of Energy, Office of Science, Office of High Energy Physics under Award Number DE-SC0014329. C-HC acknowledges support as a MultiDark Fellow. C-HC also acknowledges support from the Spanish MICINNs Consolider-Ingenio 2010 Programme under grant MultiDark CSD2009-00064, MINECO Centro de Excelencia Severo Ochoa Programme under grant SEV-2012-0249 and grant AYA2014-60641-C2-1-P.

\section{REFERENCES}

Alam S. et al., 2016, MNRAS, preprint (arXiv:1607.03155)

Anderson L. et al., 2012, MNRAS, 427, 3435

Anderson L. et al., 2014, MNRAS, 441, 24

Ballinger W. E., Peacock J. A., Heavens A. F., 1996, MNRAS, 282, 877

Beutler F. et al., 2011, MNRAS, 416, 3017

Beutler F. et al., 2014, MNRAS, 443, 1065

Beutler F., Blake C., Koda J., Marin F., Seo H. J., Cuesta A. J., Schneider D. P., 2016, MNRAS, 455, 3230

Beutler F. et al., 2016, MNRAS, preprint (arXiv:1607.03150) (B16)

Bianchi D., Gil-Marin H., Ruggeri R., Percival W. J., 2015, MNRAS, 453, L11

Blake C., Glazebrook K., 2003, ApJ, 594, 665

Blake C. et al., 2011, MNRAS, 418, 1707

Blazek J., McEwen J. E., Hirata C. M., 2016, Phys. Rev. Lett., 116, 121303

Bolton A. S. et al., 2012, AJ, 144, 144

Burden A., Percival W. J., Manera M., Cuesta A. J., Magana M. V., Ho S., 2014, MNRAS, 445, 3152

Cole S. et al., 2005, MNRAS, 362, 505

Crocce M., Scoccimarro R., 2006, Phys. Rev. D, 73, 063520

Crocce M., Scoccimarro R., 2008, Phys. Rev. D, 77, 023533

Dalal N., Pen U.-L., Seljak U., 2010, J. Cosmol. Astropart. Phys., 11, 007

Dawson K. S. et al., 2013, AJ, 145, 10
Doi M. et al., 2010, AJ, 139, 1628

Eisenstein D. J., 2003, in Brown M. J. I., Dey A., eds, ASP Conf. Ser. Vol. 280, Next Generation Wide Field Multi-Object Spectroscopy. Astron. Soc. Pac., San Francisco, p. 35

Eisenstein D. J., Hu W., 1998, ApJ, 496, 605

Eisenstein D. J. et al., 2005, ApJ, 633, 560

Eisenstein D. J., Seo H. J., White M. J., 2007a, ApJ, 664, 660

Eisenstein D. J., Seo H. J., Sirko E., Spergel D., 2007b, ApJ, 664, 675

Eisenstein D. J. et al., 2011, AJ, 142, 72

Feldman H. A., Kaiser N., Peacock J. A., 1994, ApJ, 426, 23

Font-Ribera A., McDonald P., Mostek N., Reid B. A., Seo H.-J., Slosar A., 2014, J. Cosmol. Astropart. Phys., 05, 023

Fukugita M., Ichikawa T., Gunn J. E., Doi M., Shimasaku K., Schneider D. P., 1996, AJ, 111, 1748

Gil-Marin H. et al., 2016, MNRAS, 460, 4210

Grieb et al., 2016, MNRAS, preprint (arXiv:1607.03143)

Gunn J. E. et al., 1998, AJ, 116, 3040

Gunn J. E. et al., 2006, AJ, 131, 2332

Guo H., Zehavi I., Zheng Z., 2012, ApJ, 756, 127

Hamilton A. J. S., 2000, MNRAS, 312, 257

Hartlap J., Simon P., Schneider P., 2007, A\&A, 464, 399

Hu W., Haiman Z., 2003, Phys. Rev. D, 68, 063004

Hu W., White M., 1996, ApJ, 471, 30

Jain B., Bertschinger E., 1994, ApJ, 431, 495

Jing Y. P., 2005, ApJ, 620, 559

Kaiser N., 1987, MNRAS, 227, 1

Kazin E. A. et al., 2014, MNRAS, 441, 3524

Kitaura F. S., Yepes G., Prada F., 2014, MNRAS, 439, 21

Kitaura F.-S. et al., 2016, MNRAS, 456, 4156

Klypin A., Yepes G., Gottlober S., Prada F., Hess S., 2016, MNRAS, 457, 4340

Linder E. V., 2005, Phys. Rev. D, 72, 043529

Manera M. et al., 2013, MNRAS, 428, 1036

Manera M. et al., 2015, MNRAS, 447, 437

Matsubara T., 2008a, Phys. Rev. D, 77, 063530

Matsubara T., 2008b, Phys. Rev. D, 78, 083519 [Erratum Phys. Rev. D, 2008, 78, 109901]

Mehta K. T., Seo H.-J., Eckel J., Eisenstein D. J., Metchnik M., Pinto P., Xu X., 2011, ApJ, 734, 94

Meiksin A., White M., Peacock J. A., 1999, MNRAS, 304, 851

Ngan W., Harnois-Déraps J., Pen U.-L., McDonald P., MacDonald I., 2012, MNRAS, 419, 2949

Nusser A., Davis M., 1994, ApJ, 421, L1

Padmanabhan N., White M., 2009, Phys. Rev. D, 80, 063508

Padmanabhan N., Xu X., Eisenstein D. J., Scalzo R., Cuesta A. J., Mehta K. T., Kazin E., 2012, MNRAS, 427, 2132

Peebles P. J. E., Yu J. T., 1970, ApJ, 162, 815

Percival W. J. et al., 2001, MNRAS, 327, 1297

Percival W. J. et al., 2014, MNRAS, 439, 2531

Planck Collaboration XIII, 2016, A\&A, 594, A13

Reid B. A., Seo H. J., Leauthaud A., Tinker J. L., White M., 2014, MNRAS, 444,476

Reid B. et al., 2016, MNRAS, 455, 1553

Rodriguez-Torres S. A. et al., 2016, MNRAS, 460, 1173

Ross A. J. et al., 2012, MNRAS, 424, 564

Ross A. J., Samushia L., Howlett C., Percival W. J., Burden A., Manera M., 2015, MNRAS, 449, 835

Ross A. J., Percival W. J., Manera M., 2015, MNRAS, 451, 1331

Ross A. J. et al., 2016, MNRAS, 464, 1168

Sanchez A. G. et al., 2016, MNRAS, preprint (arXiv:1607.03146)

Satpathy S. et al., 2016, MNRAS, preprint (arXiv:1607.03148)

Schmittfull M., Feng Y., Beutler F., Sherwin B., Chu M. Y., 2015, Phys. Rev. D, 92, 123522

Scoccimarro R., 2015, Phys. Rev. D, 92, 083532

Sefusatti E., Crocce M., Scoccimarro R., Couchman H., 2016, MNRAS, 460,3624

Seo H. J., Eisenstein D. J., 2003, ApJ, 598, 720

Seo H.-J., Eisenstein D. J., 2005, ApJ, 633, 575 
Seo H. J., Eisenstein D. J., 2007, ApJ, 665, 14

Seo H. J., Siegel E. R., Eisenstein D. J., White M., 2008, ApJ, 686, 13

Seo H.-J. et al., 2010, ApJ, 720, 1650

Seo H. J., Beutler F., Ross A. J., Saito S., 2016, MNRAS, 460, 2453

Sherwin B. D., Zaldarriaga M., 2012, Phys. Rev. D, 85, 103523

Shoji M., Jeong D., Komatsu E., 2009, ApJ, 693, 1404

Smee S. et al., 2013, AJ, 146, 32

Smith J. A. et al., 2002, AJ, 123, 2121

Springel V., 2005, MNRAS, 364, 1105

Sunyaev R. A., Zeldovich Y. B., 1970, Ap\&SS, 7, 3

Taruya A., Nishimichi T., Saito S., Hiramatsu T., 2009, Phys. Rev. D, 80, 123503

Taruya A., Nishimichi T., Saito S., 2010, Phys. Rev. D, 82, 063522

Tojeiro R. et al., 2014, MNRAS, 440, 2222

Tseliakhovich D., Hirata C., 2010, Phys. Rev. D, 82, 083520

Vargas-Magaña M., Ho S., Fromenteau S., Cuesta A. J., 2015, preprint (arXiv:1509.06384)

Vargas-Magaña M. et al., 2016, MNRAS, preprint (arXiv:1610.03506)

White M., 2015, MNRAS, 450, 3822

Wilson M. J., Peacock J. A., Taylor A. N., de la Torre S., 2015, preprint (arXiv: 1511.07799)

Xu X., Padmanabhan N., Eisenstein D. J., Mehta K. T., Cuesta A. J., 2012, MNRAS, 427, 2146

Yamamoto K., Nakamichi M., Kamino A., Bassett B. A., Nishioka H., 2006, PASJ, 58, 93

Yoo J., Seljak U., 2015, MNRAS, 447, 1789

Yoo J., Dalal N., Seljak U., 2011, J. Cosmol. Astropart. Phys., 07, 018

\section{APPENDIX A: NGC VERSUS SGC}

Fig. A1 shows the best-fitting isotropic power spectrum models compared to the monopole measurements for the three redshift bins pre- and post-reconstruction. These plots are similar to Fig. 14, but here we separate the SGC and NGC components.

\section{APPENDIX B: COMBINING NGC AND SGC}

We combine the NGC and SGC measurements in Figs 10 and 14 using

$C_{\mathrm{NGC}+\mathrm{SGC}}^{-1}=C_{\mathrm{NGC}}^{-1}+C_{\mathrm{SGC}}^{-1}$

and

$C_{\mathrm{NGC}+\mathrm{SGC}}^{-1} P(k)=C_{\mathrm{NGC}}^{-1} P_{\mathrm{NGC}}(k)+C_{\mathrm{SGC}}^{-1} P_{\mathrm{SGC}}(k)$,

where $C$ is the covariance matrix measured from the MultiDarkPatchy mock catalogues.

\section{APPENDIX C: COMPARISON TO THE FISHER MATRIX FORECASTS}

As summarized in Section 10.3, here we compare our BOSS measurements with the original Fisher forecasts for the survey (Seo \& Eisenstein 2007; Eisenstein et al. 2011) to see whether BOSS has performed as expected. We start with the isotropic constraints. Using the MultiDark-Patchy mock catalogues we can construct the correlation matrix for the angle averaged distance constraint $D_{V}$ :

$R=\left(\begin{array}{ccc}1 & & \\ 0.392 & 1 & \\ 0.033 & 0.437 & 1\end{array}\right)$.
This matrix suggests that there is almost no correlation between the low- and high-redshift bin. We can now derive a combined isotropic constraint as

$\sigma_{\alpha, \text { comb iso }}=\frac{1}{\sqrt{\sum C_{i j}^{-1}}}$,

where $C^{-1}$ is the inverse covariance matrix, which can be obtained by combining the correlation matrix above with the measurement uncertainties in Table 3 . We obtain a combined constraint of $\sigma_{\alpha \text {, comb iso }}=0.00643$ (post-reconstruction). Ignoring the middleredshift bin and assuming that the high- and low-redshift bins are not correlated, we find $\sigma_{\alpha, \text { comb iso }}^{\prime}=1 / \sqrt{1 / \sigma_{z 1}^{2}+1 / \sigma_{z 3}^{2}}=0.00656$, which is close to the former value. This result suggests that the middle-redshift bin does not contain much additional information. This is the combined error from our isotropic fits to the monopole - the error on $\alpha$ from the anisotropic fit, similarly computed, is a bit worse at $\sigma_{\alpha \text {,comb aniso }}=0.0070$. Generally we would expect $\sigma_{\alpha, \text { comb aniso }}$ to correspond more directly to the optimally averaged dilation factor error coming out of the Fisher matrix projections (Seo \& Eisenstein 2007). The difference must enter through the isotropic versus anisotropic fitting details, as the error on the $D_{V}$ factor $\alpha_{\|}^{1 / 3} \alpha_{\perp}^{2 / 3}$ is only $\sim 1$ per cent larger than an optimally weighted dilation factor error.

BOSS was originally projected to achieve measurements of $D_{\mathrm{A}}(z)$ and $H(z)$ to 1.0 and 1.8 per cent, respectively, at $z=0.35$, and 1.0 and 1.7 per cent at $z=0.6$ (Eisenstein et al. 2011). These original projections combine to 0.48 per cent overall isotropic error (FontRibera et al. 2014), which is a factor of 1.44 smaller than our measurement of 0.70 per cent (see above, where we use the aggregated anisotropic result). This is a larger discrepancy than we are happy to accept without explanation (equivalent to a factor 0.48 reduction in survey area, for example).

Statistical fluctuations are one potential source of discrepancy between predicted and achieved errors. For this reason we might at first think it is better to look at results averaged over many mocks for a more accurate gauge of the survey performance. By this criterion the error discrepancy is actually quite a bit worse, at 1.82 times expected (i.e. combined $\alpha$ error for mocks of 0.88 per cent in Table 2). However, as discussed in Section 8.3, we believe the large errors in the mocks are due to excessively damped BAO in the mocks, and therefor do not give a realistic survey expectation. An alternative way to take randomness out of the achieved BAO errors is to estimate the errors by taking approximate second derivatives of $\chi^{2}$ with respect to model parameters around the pre-survey expected model, in contrast to our standard estimate that fully marginalizes over all the parameters. To be concrete: with $\chi^{2}=[\boldsymbol{d}-\boldsymbol{t}(\boldsymbol{\theta})]^{t} \mathbf{C}^{-1}[\boldsymbol{d}-\boldsymbol{t}(\boldsymbol{\theta})]$, where $\boldsymbol{d}$ is the data vector (band power measurement) and $\boldsymbol{t}(\boldsymbol{\theta})$ the theory predictions for it as a function of parameter vector $\boldsymbol{\theta}$, our standard way of estimating errors on parameter $\theta_{i}$ is to use the likelihood formula $L(\boldsymbol{\theta}) \propto \exp \left[-\chi^{2}(\boldsymbol{\theta}) / 2\right]$ to compute $\sigma_{\theta_{i}}=\left\langle\left(\theta_{i}-\bar{\theta}_{i}\right)^{2}\right\rangle^{1 / 2}$, with $\bar{\theta}_{i}=\left\langle\theta_{i}\right\rangle$, by integrating over all $\boldsymbol{\theta}$ [e.g. by Markov chain Monte Carlo (MCMC)]. An alternative method is to assume the likelihood is Gaussian in $\boldsymbol{\theta}$ around some Taylor expansion point and invert the second derivative matrix $\frac{\mathrm{d}^{2} \chi^{2}}{\mathrm{~d} \theta_{i} \mathrm{~d} \theta_{j}}$ to find the implied covariance matrix for the parameters. The standard choice of expansion point would be the maximum likelihood point, which is equivalent to the exact likelihood integration in the small-error limit, however, choosing this point (or doing the exact integration) makes the results sensitive to the actual data points (i.e. the maximum likelihood parameter vector is $\boldsymbol{\theta}_{\max L}=\boldsymbol{\theta}_{\max L}(\boldsymbol{d})$ ), which can push you into an area of parameter 

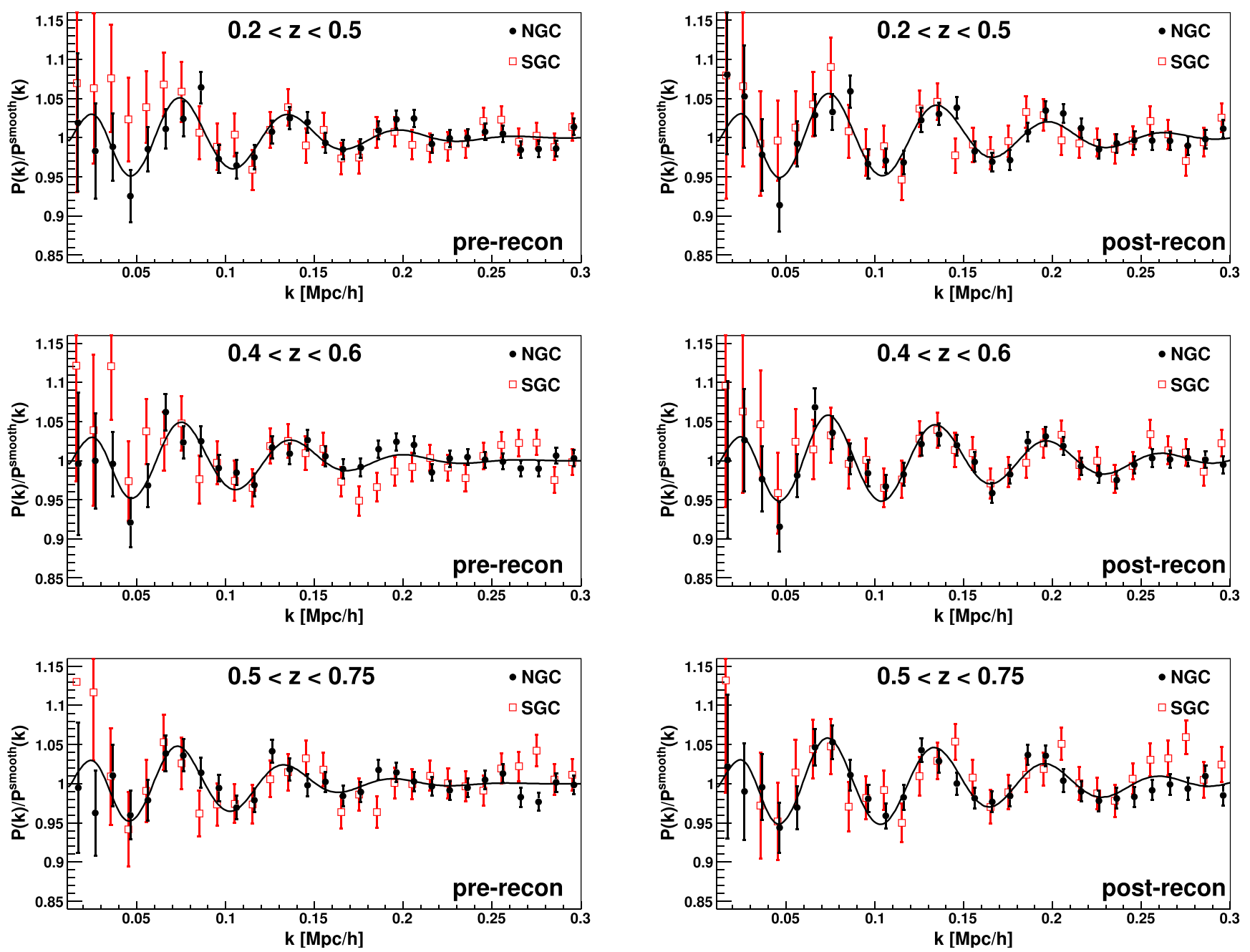

Figure A1. The best-fitting results for the isotropic BAO analysis pre-reconstruction (left) and post-reconstruction (right). The NGC (black circles) and SGC (open red squares) measurements are displayed relative to the smooth power spectrum (see equation 50). After dividing by the smooth power spectrum, the best-fitting model for the NGC and SGC is the same (solid black line). The uncertainties are the diagonal of the covariance matrix. The covariance matrix has significant non-diagonal terms at large wavenumbers, which are larger in the pre-reconstruction case. The best-fitting parameters and $\chi^{2}$ are included in the lower part of Table 3 .

space with bigger or smaller errors (e.g. because of statistical fluctuations in the apparent shape of the BAO feature). On the other hand, expanding around a prior expected $\boldsymbol{\theta}$, and approximating the second derivative matrix as $\frac{\mathrm{d} t^{t}}{\mathrm{~d} \theta_{i}} \mathbf{C}^{-1} \frac{\mathrm{d} t}{\mathrm{~d} \theta_{j}}$ gives an error manifestly insensitive to the data points (i.e. $\boldsymbol{d}$ ), only reflecting the covariance matrix and the structure of the theory, i.e., this is like the Fisher matrix estimate, except with a predicted $\mathbf{C}$ replaced with the achieved one. For this comparison we use a slightly different broad-band model that allows for more arbitrary fluctuations in BAO amplitude and damping (in this model, we use interpolation points in $k$ as the free parameters, including both an additive function and function that multiplies the BAO wiggles - an advantage of this model is that the predicted power is linear in all parameters except the BAO distance scale, so that we can perform exact marginalization over all the nuisance parameters essentially instantly, making the fits very fast). The alternative model gives an error of 0.68 per cent (i.e. very similar to our quoted 0.66 per cent, where we use only the upper and lower redshift bins here, and fit the monopole only - this is also close to the 0.70 per cent we found from the anisotropic fit) with full marginalization, and also 0.68 percent when expanding around the a priori expected model (e.g. with BAO damping given by 50 per cent reconstruction of the non-linear damping factors of Seo \& Eisenstein 2007). The similarity of these results suggests that we are not seeing significant random fluctuations in the BAO measurement error relative to expected (note that we do use the lower bias mentioned below here, which has a small error driven by the broad-band power spectrum and therefore should not contribute randomness to the $\mathrm{BAO}$ error).

The next level of comparison is to estimate $\mathrm{BAO}$ errors given predictions for the Fisher band power errors based on the survey area, galaxy number density and bias as a function of redshift (i.e. not using the Seo \& Eisenstein 2007 code). After computing the Fisher band power errors, we derive BAO errors as discussed above, by taking derivatives of $\chi^{2}$, using the same theory $(\boldsymbol{t}(\boldsymbol{\theta}))$ that we use to fit the data but now using the Fisher band power errors for $\mathbf{C}$. Given the pre-survey nominal bias $b(z)=1.7 D(0) / D(z)$ and number densities (Font-Ribera et al. 2014) over $10000 \mathrm{deg}^{2}$, we find that band power predictions propagate to 0.59 per cent distance error, still 23 percent over the original expectation but closer than the achieved $\sim 0.68$ per cent. [To be clear: the difference between 
the original projection of 0.48 and 0.59 percent is the difference between using the Seo \& Eisenstein (2007) code and a new projection of the expected Fisher band power errors given the original survey parameters, propagated to BAO errors by direct application of our BAO fitting theory instead of the approximations of Seo \& Eisenstein (2007).]

It turns out that the measured bias is $\sim 0.8$ times expected, producing larger fractional band power errors. Accounting for this in the Fisher band power prediction results in a predicted BAO measurement uncertainty of 0.68 per cent. Further accounting for small differences between expected and achieved area and number density brings this to 0.71 per cent, essentially equivalent to the measured value. On the other hand, we know that the measured errors could be 3 per cent smaller if we had infinite mocks (equation 26), and it appears in Figs 1 and 2 that the power in the mocks is sometimes too high relative to the measurement, which we estimate could be wrongly adding another $\sim 3$ per cent to the measured errors, i.e. running infinite mocks with correct power would result in measured errors $\sim 6$ per cent smaller than we quote. Putting these pieces together suggests that the band power errors estimated from the mocks are, if anything, a little bit better than one would expect based on observed survey volume, numbers and bias.

So with $\sim 20$ per cent underachievement $(0.71 / 0.59)$ relative to pre-survey expectations accounted for by bias, number of galaxies and sky area (mostly the lower than expected bias) we are left to explain the remaining $\sim 23$ per cent $(0.59 / 0.48)$ discrepancy between predictions based on applying our BAO fitting apparatus to the Fisher band power error predictions and based on the approximations in the code of Seo \& Eisenstein (2007) [i.e. the Seo \& Eisenstein (2007) projection is optimistic relative to both data and the Fisher predicted band powers]. The two projections (Seo \& Eisenstein 2007 versus fitting Fisher band powers) nominally make essentially the same assumptions, i.e. are supposed to be just different ways of numerically evaluating the same basic Fisher matrix equation, so there is no reason to expect this kind of difference, but the Seo \& Eisenstein (2007) code employs approximations and calibration of the signal strength that make a very direct comparison difficult. Surprisingly, however, it seems that the difference is mostly accounted for by the difference between our fiducial cosmology and the WMAP3 cosmology hard-coded in the Seo \& Eisenstein (2007) code. The Fisher band-power BAO error based on the WMAP3 model, at fixed total number of observed galaxies and observed power amplitude, is 0.51 per cent - within the margin of fine details of the 0.48 per cent of Seo \& Eisenstein (2007). A 16 per cent $(0.59 / 0.51)$ change between cosmologies may seem surprising, but recall that the WMAP3 cosmology had $\sim 12$ per cent higher baryon to CDM density ratio than the current standard, with correspondingly larger BAO signal amplitude, which very simply accounts for most of the difference.

Now we want to look at the anisotropic constraints in the form of $\alpha_{\perp}$ and $\alpha_{\|}$. The combined covariance matrix for $\alpha_{\perp}$ and $\alpha_{\|}$is

$$
\begin{aligned}
& C_{z 1+z 3}=\left[C_{z 1}^{-1}+C_{z 3}^{-1}\right]^{-1} \\
& =\left(\begin{array}{cc}
\sigma_{\alpha_{\|}}^{2} & r \sigma_{\alpha_{\perp}} \sigma_{\alpha_{\|}} \\
r \sigma_{\alpha_{\perp}} \sigma_{\alpha_{\|}} & \sigma_{\alpha_{\perp}}^{2}
\end{array}\right) \\
& =10^{-4}\left(\begin{array}{lll}
3.1 & -0.83-0.83 & 1.2
\end{array}\right) .
\end{aligned}
$$

This produces a 1.76 percent constraint on $\alpha_{\|}(\propto 1 / H)$ and a 1.09 per cent constraint on $\alpha_{\perp}\left(\propto D_{\mathrm{A}}\right)$ with a correlation of $r=-0.42$ (post-reconstruction). Compared to the two 1 per cent $D_{\mathrm{A}}$ measure- ments originally predicted by Eisenstein et al. (2011), and 1.7 and 1.8 per cent predicted $H$ measurements, the measurement errors are 1.54 and 1.42 times expected for $D_{\mathrm{A}}$ and $H$, respectively. This is similar to the 1.44 times expected that we found for $\alpha$. We speculate that the reduction in signal due to bias and cosmology leads to more degradation of the measurement in the transverse than radial direction because the radial direction is boosted by redshift-space distortions.

${ }^{1}$ Institute of Cosmology and Gravitation, Dennis Sciama Building, University of Portsmouth, Portsmouth PO1 3FX, UK

${ }^{2}$ Lawrence Berkeley National Lab, 1 Cyclotron Rd, Berkeley, CA 94720, USA

${ }^{3}$ Department of Physics and Astronomy, Ohio University, $251 B$ Clippinger Labs, Athens, OH 45701, USA

${ }^{4}$ Department of Physics, Ohio State University, 140 West 18th Avenue, Columbus, $\mathrm{OH}$ 43210, USA

${ }^{5}$ Kavli Institute for the Physics and Mathematics of the Universe (WPI), The University of Tokyo Institutes for Advanced Study, The University of Tokyo, Kashiwa, Chiba 277-8583, Japan

${ }^{6}$ Max-Planck-Institut für Astrophysik, Karl-Schwarzschild-Strasse 1, D-85740 Garching bei München, Germany

${ }^{7}$ Department of Physics and Astronomy, University of Utah, 115 South 1400 East, Salt Lake City, UT 84112, USA

${ }^{8}$ National Optical Astronomy Observatory, $950 \mathrm{~N}$ Cherry Ave, Tucson, AZ 85719, USA

${ }^{9}$ Instituto de Física Teórica (UAM/CSIC), Universidad Autónoma de Madrid, Cantoblanco, E-28049 Madrid, Spain

${ }^{10}$ Leibniz-Institut für Astrophysik Potsdam (AIP), An der Sternwarte 16, D-14482 Potsdam, Germany

${ }^{11}$ Institut de Ciències del Cosmos (ICCUB), Universitat de Barcelona (IEEC-UB), Martí i Franquès 1, E-08028 Barcelona, Spain

${ }^{12}$ Harvard-Smithsonian Center for Astrophysics, 60 Garden St., Cambridge, MA 02138, USA

${ }^{13}$ Universitäts-Sternwarte München, Ludwig-Maximilians-Universität München, Scheinerstraße 1, D-81679 München, Germany

${ }^{14}$ Max-Planck-Institut für extraterrestrische Physik, Postfach 1312, Giessenbachstr., D-85741 Garching, Germany

${ }^{15}$ Department of Astronomy, University of California, Berkeley, CA 94720 , USA

${ }^{16}$ Department of Physics, University of California, Berkeley, CA 94720, USA

${ }^{17}$ Campus of International Excellence UAM+CSIC, Cantoblanco, E-28049 Madrid, Spain

${ }^{18}$ Instituto de Astrofísica de Andalucía (CSIC), Glorieta de la Astronomía, E-18080 Granada, Spain

${ }^{19}$ Institute for Astronomy, University of Edinburgh, Royal Observatory, Edinburgh EH9 3HJ, UK

${ }^{20}$ Department of Astronomy and Astrophysics, The Pennsylvania State University, University Park, PA 16802, USA

${ }^{21}$ Institute for Gravitation and the Cosmos, The Pennsylvania State University, University Park, PA 16802, USA

${ }^{22}$ Brookhaven National Laboratory, Upton, NY 11973, USA

${ }^{23}$ Center for Cosmology and Particle Physics, Department of Physics, New York University, 4 Washington Place, New York, NY 10003, USA

${ }^{24}$ School of Physics and Astronomy, University of St. Andrews, St Andrews, Fife KY16 9SS, UK

${ }^{25}$ Instituto de Fisica, Universidad Nacional Autonoma de Mexico, Apdo. Postal 20-364, Mexico

This paper has been typeset from a $\mathrm{T}_{\mathrm{E}} \mathrm{X} / \mathrm{LT}_{\mathrm{E}} \mathrm{X}$ file prepared by the author. 